\title{
Spinning AdS propagators
}

\section{Miguel S. Costa, Vasco Gonçalves and João Penedones}

Centro de Fúsica do Porto, Departamento de Física e Astronomia, Faculdade de Ciências da Universidade do Porto, Rua do Campo Alegre 687, 4169-007 Porto, Portugal

E-mail: miguelc@fc.up.pt, vasco.dfg@gmail.com, jpenedones@fc.up.pt

ABSTRACT: We develop the embedding formalism to describe symmetric traceless tensors in Anti-de Sitter space. We use this formalism to construct the bulk-to-bulk propagator of massive spin $J$ fields and check that it has the expected short distance and massless limits. We also find a split representation for the bulk-to-bulk propagator, by writing it as an integral over the boundary of the product of two bulk-to-boundary propagators. We exemplify the use of this representation with the computation of the conformal partial wave decomposition of Witten diagrams. In particular, we determine the Mellin amplitude associated to AdS graviton exchange between minimally coupled scalars of general dimension, including the regular part of the amplitude.

KEYwords: Higher Spin Gravity, AdS-CFT Correspondence

ArXiv EPRINT: 1404.5625 


\section{Contents}

1 Introduction $\quad 2$

2 Embedding formalism for AdS 3

$\begin{array}{lll}3 & \text { AdS propagators of spinning particles } & 6\end{array}$

$\begin{array}{lll}3.1 \text { Bulk-to-bulk propagator } & 7\end{array}$

$\begin{array}{lll}3.1 .1 & \text { Spin } 1 & 10\end{array}$

$\begin{array}{lll}3.1 .2 & \text { Spin } 2 & 10\end{array}$

3.2 Bulk-to-boundary propagator 11

$\begin{array}{ll}3.3 \text { Short distance limit } & 12\end{array}$

3.4 Massless limit 13

$\begin{array}{lll}3.4 .1 & \text { Graviton } & 14\end{array}$

4 Split representation of AdS propagators $\quad 14$

4.1 Spin $J$ harmonic functions in AdS 14

$\begin{array}{lll}4.2 & \text { Split representation } & 16\end{array}$

$\begin{array}{lll}4.3 & \text { Spin } 1 & 19\end{array}$

$\begin{array}{lll}4.4 & \text { Spin } 2 & 19\end{array}$

$\begin{array}{lll}\text { 4.4.1 Graviton propagator } & 21\end{array}$

5 Three-point function $\quad 21$

6 Four-point function $\quad \mathbf{2 5}$

6.1 Example: AdS graviton exchange 27

$\begin{array}{lll}\text { 6.1.1 Mellin amplitude } & 28\end{array}$

$\begin{array}{lll}7 & \text { Concluding remarks } & 31\end{array}$

A Harmonic functions in flat space $\quad 32$

$\begin{array}{ll}\text { B Embedding space operations } & 34\end{array}$

C Split representation of the bulk-to-bulk propagator 35

$\begin{array}{ll}\text { D AdS harmonic functions } & 37\end{array}$

$\begin{array}{ll}\text { E Computation of partial amplitude } & 41\end{array}$ 


\section{Introduction}

Higher spin fields play an important role in all known examples of the AdS/CFT duality [1]. In the case of the $\mathcal{N}=4 \mathrm{SYM}$ there are massive string states in AdS, which must be taken into account at finite 't Hooft coupling $\lambda$, and whose effect appears at infinite coupling in the form of $1 / \sqrt{\lambda}$ corrections. More recently there has been a resurgence of higher spin gauge theories in $A d S_{4}$ [2-5], conjectured to be dual to three-dimensional CFTs like the $O(N)$ vector model [6], the Gross-Neveu model [7] or certain large $N$ Chern-Simons theories [8]. In all these recent cases, computations involving AdS higher spin fields pose additional technical challenges. The goal of this paper is to develop a formalism to deal with tensor fields in AdS, that makes computations almost as simple as those with scalar fields.

More specifically, we shall develop the embedding formalism for treating massive symmetric traceless AdS tensor fields with $J$ indices (or spin $J$ fields, for short), but some of the methods here developed should be extendable to antisymmetric tensors or mixed symmetry tensors. The basic idea of the embedding formalism is that fields in Euclidean $A d S_{d+1}$ space, or their $C F T_{d}$ dual operators, can be expressed in terms of fields in an embedding Minkowski space $\mathbb{M}^{d+2}$. The action of the $A d S_{d+1}$ isometry group, or of the conformal group $\mathrm{SO}(d+1,1)$, can then be realised as the group of linear Lorentz transformations. This fact has been explored in many places in the literature to simplify computations, including computations of correlation functions of higher spin fields [9-12], of conformal blocks for external operators with spin [13, 14], and of Witten diagrams to derive Feynman rules in Mellin space [15-17], to name a few.

We shall start, in section 2, by introducing the basic definitions that allow us to describe AdS fields in the embedding formalism, including the representation of differential operators such as the Laplacian. As a first application of the formalism, we compute in section 3 the bulk-to-bulk propagator of a massive spin $J$ field in AdS. Explicit expressions for the scalar and spin 1 cases are known for a long time, while the expressions for the massive spin 2 and p-form cases are known only more recently [18]. The new propagator has the required short distance behaviour derived in [19] using zeta function regularisation. It also reproduces the known form of the vector propagator, as well as the traceless part of the graviton propagator in the massless limit, as given in [20]. In general it is known that the bulk-to-bulk propagator is closely related to the product of two bulk-to-boundary propagators integrated over a common boundary point [21, 22]. In section 4 we make this relation precise by deriving a split representation for spin $J$ propagators in AdS. We also consider the case of the graviton propagator whose split representation has generated some discussion in the literature [23, 24]. Taking one of the bulk points to the boundary, the spin $J$ propagator also defines the bulk-to-boundary propagator for this field. As an application of this result we derive in section 5 the relation between the AdS local coupling of two scalar fields and one spin $J$ field, and the OPE coefficient of the dual CFT operators. Finally, in section 6 we make use of this split representation to derive the conformal block expansion of four-point correlation functions computed via Witten diagrams. In particular, we determine the Mellin amplitude associated to AdS graviton 


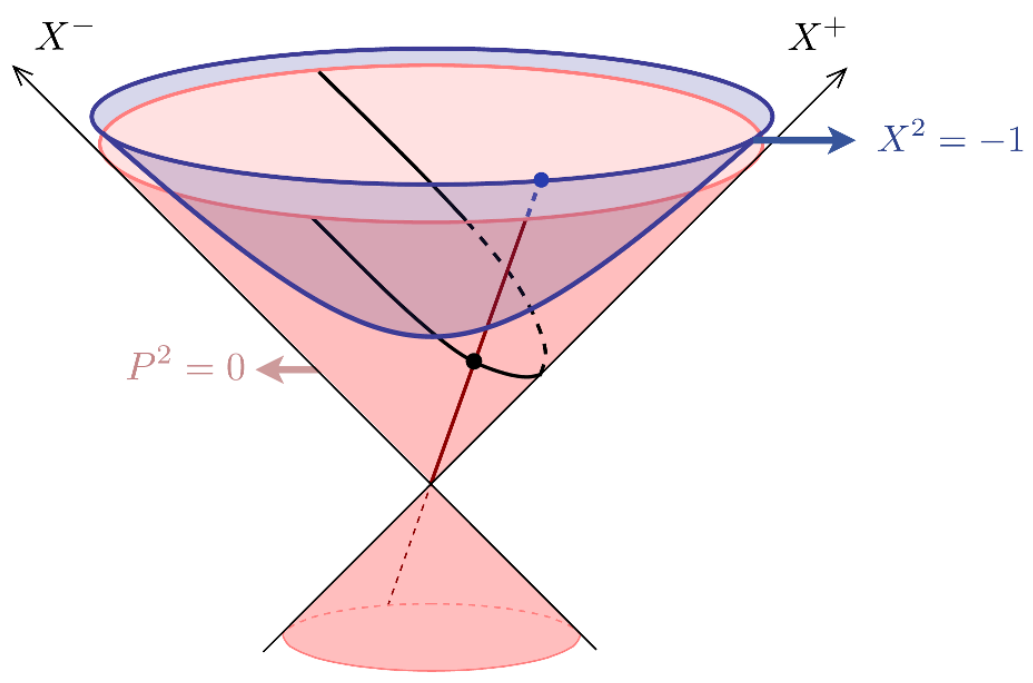

Figure 1. Euclidean AdS and its boundary in the embedding space. This picture shows the $A d S_{2}$ surface $X^{2}=-1$ and the identification of a boundary point (in blue) with a light ray (in red) of the light cone $P^{2}=0$, which intersects the Poincaré section on a (black) point.

exchange between minimally coupled massive scalars in general spacetime dimension. A number of technical computations are left to appendices.

\section{Embedding formalism for AdS}

In this paper we consider tensor fields in Euclidean $(d+1)$-dimensional Anti de Sitter space $\operatorname{AdS}_{d+1}$. Obviously this is just the $(d+1)$-dimensional hyperbolic space. Our expressions can be Wick-rotated to Minkowski signature, provided one is careful with the $i \epsilon$ prescription (see $[25,26]$ for some details). In this section we introduce notation and develop the embedding formalism to treat tensor fields in $\mathrm{AdS}_{d+1}$. We shall see how the use of this formalism simplifies computations considerably, making conformal invariance manifest at all time, just like for tensor fields in $d$-dimensional CFTs. This simple idea has been used before (e.g. [27, 28]) and is also known has tractor calculus $[29,30]$.

Euclidean $\mathrm{AdS}_{d+1}$ space can be defined by the set of future directed unit vectors,

$$
X^{2}=-1, \quad X^{0}>0
$$

in $(d+2)$-dimensional Minkowski space $\mathbb{M}^{d+2}$. As it is well known, the isometry group of $\operatorname{AdS}_{d+1}$ is the $d$-dimensional conformal group $\mathrm{SO}(d+1,1)$. This group acts linearly on the embedding space $\mathbb{M}^{d+2}$, and its action is interior to points on the hyperboloid $X^{2}=-1$. A simple example is that of $\operatorname{AdS}_{d+1}$ written in Poincaré coordinates $x^{\mu}=\left(z, y^{a}\right)$, with $y$ a $d$-dimensional vector. In this case AdS points are parameterized as

$$
X=\frac{1}{z}\left(1, z^{2}+y^{2}, y^{a}\right),
$$


where we used light cone coordinates

$$
X^{A}=\left(X^{+}, X^{-}, X^{a}\right)
$$

with metric

$$
X \cdot X=\eta_{A B} X^{A} X^{B}=-X^{+} X^{-}+\delta_{a b} X^{a} X^{b} .
$$

Here and below, we use capital letters to denote embedding space indices in $\mathbb{M}^{d+2}$, lower case letters to denote indices in $\mathbb{R}^{d}$, and greek letters to denote $\mathrm{AdS}_{d+1}$ indices.

AdS boundary points can be obtained by sending some of the $X$ coordinates to infinity. In this limit the hyperboloid approaches the light cone, so that a given specific point at infinity in the hyperboloid approaches one light ray. This allows for the identification of the AdS boundary with light rays, according to

$$
P^{2}=0, \quad P \equiv \lambda P, \quad \lambda \in \mathbb{R} .
$$

For example, for the Poincaré patch considered above, boundary points are parameterised by

$$
P=\left(1, y^{2}, y^{a}\right)
$$

figure 1 summarizes this geometric picture.

We wish to establish the relation between fields in $\mathrm{AdS}_{d+1}$ and $\mathbb{M}^{d+2}$. In particular, here we will consider traceless symmetric tensors. Let us then consider a traceless symmetric tensor of $\mathbb{M}^{d+2}$ with components $H_{A_{1} \ldots A_{J}}(X)$, defined on the surface $X^{2}=-1$ and transverse to this surface,

$$
X^{A_{1}} H_{A_{1} \ldots A_{J}}(X)=0 .
$$

This defines a tensor in $\mathrm{AdS}_{d+1}$, whose components are simply obtained by the projection

$$
h_{\mu_{1} \ldots \mu_{J}}=\frac{\partial X^{A_{1}}}{\partial x^{\mu_{1}}} \cdots \frac{\partial X^{A_{J}}}{\partial x^{\mu_{J}}} H_{A_{1} \ldots A_{J}}(X)
$$

The extension of the embedding tensor $H(X)$ away from the $\mathrm{AdS}_{d+1}$ submanifold $X^{2}=-1$ is not physical. On one hand, this means that components of the tensor that are transverse to the hyperboloid, i.e. of the type

$$
H_{A_{1} \ldots A_{J}}(X)=X_{\left(A_{1}\right.} \Psi_{\left.A_{2} \ldots A_{J}\right)}(X) .
$$

are unphysical. Indeed, these components, which do not satisfy the transverse condition (2.7), have vanishing projection to $\mathrm{AdS}_{d+1}$. On the other hand, it also means that whenever we take a derivative in the embedding space, that derivative can only be tangent to the $\mathrm{AdS}_{d+1}$ submanifold.

We wish to have a more economical way of encoding $\mathrm{AdS}_{d+1}$ tensors, without having to deal with all the indices and constraints arising from the linear realization of the $\mathrm{SO}(d+1,1)$ symmetry. Let us first recall how this can be achieved in the case of $\mathbb{R}^{d}$ tensors, extensively discussed in [11]. In this case a symmetric traceless tensor with components $F_{A_{1} \cdots A_{J}}(P)$ is defined on the light-cone $P^{2}=0$ of the embedding space with the requirement that 
$F(\lambda P)=\lambda^{-\Delta} F(P)$, for $\lambda>0$, where $\Delta$ is the conformal dimension. This tensor can be encoded in the polynomial

$$
F(P, Z)=Z^{A_{1}} \ldots Z^{A_{J}} F_{A_{1} \cdots A_{J}}(P),
$$

where $Z^{2}=0$ encodes the traceless condition. To be tangent to the light-cone $P^{2}=0$ the embedding tensor must satisfy $P^{A_{1}} F_{A_{1} \cdots A_{J}}=0$, which can be implemented by requiring $F(P, Z+\alpha P)=F(P, Z)$ for any $\alpha$. In addition, we can impose the orthogonality condition $P \cdot Z=0$ because $F_{A_{1} \cdots A_{J}}=P_{\left(A_{1}\right.} \Psi_{\left.A_{2} \cdots A_{J}\right)}$ has vanishing projection into physical $\mathbb{R}^{d}$ tensors. Moving to the case of AdS symmetric traceless tensors $H_{A_{1} \ldots A_{J}}(X)$, defined on the submanifold $X^{2}=-1$, they can be encoded by $(d+1)$-dimensional polynomials as

$$
H(X, W)=W^{A_{1}} \ldots W^{A_{J}} H_{A_{1} \ldots A_{J}}(X),
$$

where $W^{2}=0=X \cdot W$. The traceless condition allows one to restrict the polynomial to the submanifold $W^{2}=0$, and the transverse condition allows for the further restriction $X \cdot W=0$. In sum, a symmetric traceless tensor can be fully encoded by a polynomial $H(X, W)$ defined on the submanifold $X^{2}+1=W^{2}=X \cdot W=0$. To recover the AdS tensor from a given polynomial we define the operator

$$
\begin{aligned}
K_{A}= & \frac{d-1}{2}\left(\frac{\partial}{\partial W^{A}}+X_{A}\left(X \cdot \frac{\partial}{\partial W}\right)\right)+\left(W \cdot \frac{\partial}{\partial W}\right) \frac{\partial}{\partial W^{A}} \\
& +X_{A}\left(W \cdot \frac{\partial}{\partial W}\right)\left(X \cdot \frac{\partial}{\partial W}\right)-\frac{1}{2} W_{A}\left(\frac{\partial^{2}}{\partial W \cdot \partial W}+\left(X \cdot \frac{\partial}{\partial W}\right)\left(X \cdot \frac{\partial}{\partial W}\right)\right) .
\end{aligned}
$$

We constructed this second order differential operator such that it is interior with respect to the submanifold $X^{2}+1=W^{2}=X \cdot W=0$ (i.e. its action on a function only depends on the value of the function on this submanifold). Moreover, it is transverse $\left(X^{A} K_{A}=\right.$ $0)$, symmetric $\left(K_{A} K_{B}=K_{B} K_{A}\right)$ and traceless $\left(K_{A} K^{A}=0\right)$, so that its action on any polynomial of $W$ will define a transverse symmetric traceless AdS tensor. To be precise, it acts as a projector since

$$
\frac{1}{J !\left(\frac{d-1}{2}\right)_{J}} K_{A_{1}} \ldots K_{A_{J}} W^{B_{1}} \ldots W^{B_{J}}=G_{\left\{A_{1}\right.}^{B_{1}} \ldots G_{\left.A_{J}\right\}}^{B_{J}}
$$

where

$$
G_{A B}=\eta_{A B}+X_{A} X_{B},
$$

is the induced AdS metric (and therefore a projector). Our convention for the index symmetrization is normalized according to

$$
G_{\left\{A_{1}\right.}^{B_{1}} \ldots G_{\left.A_{J}\right\}}^{B_{J}}=\frac{1}{J !} \sum_{\pi} G_{A_{\pi_{1}}}^{B_{1}} \ldots G_{A_{\pi_{J}}}^{B_{J}}-\text { traces }
$$

where the sum is over all permutations of the $A$ indices and we subtract the traces using the AdS metric $G_{A B}$. In particular, notice that acting on the polynomial (2.11), the projector $K_{A}$ simplifies to

$$
K_{A}=\left(\frac{d-1}{2}+W \cdot \frac{\partial}{\partial W}\right) \frac{\partial}{\partial W^{A}}
$$


because the tensor $H_{A_{1} \ldots A_{J}}$ is already traceless and transverse. It is then straightforward to show that the components of the symmetric and traceless AdS tensor in (2.11) can be recovered from its polynomial via

$$
H_{A_{1} \ldots A_{J}}(X)=\frac{1}{J !\left(\frac{d-1}{2}\right)_{J}} K_{A_{1}} \ldots K_{A_{J}} H(X, W),
$$

where $(a)_{J}=\Gamma(a+J) / \Gamma(a)$ is the Pochhammer symbol. Thus from now on we will work with polynomials that uniquely determine AdS symmetric traceless tensors.

Our main goal in this paper is to construct in a systematic way and in full generality the AdS propagator for a massive spin $J$ field. This means that we need to define an embedding differential operator that computes the AdS covariant derivative. Acting on symmetric traceless tensors encoded in polynomials of $W$, as in (2.11), the embedding differential operator that does the job is

$$
\nabla_{A}=\frac{\partial}{\partial X^{A}}+X_{A}\left(X \cdot \frac{\partial}{\partial X}\right)+W_{A}\left(X \cdot \frac{\partial}{\partial W}\right) .
$$

As necessary, this operator is interior to the submanifold $X^{2}+1=W^{2}=X \cdot W=$ 0 and transverse $\left(X^{A} \nabla_{A}=0\right)$. With the help of this differential operator and of the projector (2.12), we can compute the divergence of a tensor by

$$
(\nabla \cdot H)(X, W)=\frac{1}{J\left(\frac{d-3}{2}+J\right)} \nabla \cdot K H(X, W) .
$$

The left hand side of this equation is the polynomial whose projection to AdS gives the divergence $D_{\mu_{1}} h_{\mu_{2} \ldots \mu_{J}}^{\mu_{1}}$. Instead, in the right hand side we freed first one embedding index acting with $K_{A}$, and then contracted it with the embedding differential operator $\nabla_{A}{ }^{1}$

The Laplacian of a tensor field in AdS, $\nabla^{2} H$, can be simply recovered from the polynomial

$$
\left(\nabla^{2} H\right)(X, W)=\nabla \cdot \nabla H(X, W),
$$

which, after projection to AdS, computes $D^{\nu} D_{\nu} h_{\mu_{1} \ldots \mu_{J}}$.

The embedding space can also be used to compute covariant derivatives of more general tensors (with open indices). Given an embedding tensor $T_{A_{1} \ldots A_{n}}$ obeying the transversality condition (2.7), its covariant derivative is simply given by

$$
\nabla_{B} T_{A_{1} \ldots A_{n}}(X)=G_{B}^{C} G_{A_{1}}^{C_{1}} \ldots G_{A_{n}}^{C_{n}} \frac{\partial}{\partial X^{C}} T_{C_{1} \ldots C_{n}}(X),
$$

where the projector $G_{B}{ }^{C}$ is the AdS metric given in (2.14).

\section{AdS propagators of spinning particles}

Let us first recall some basic results on particles with spin 1 and 2. A massive spin 1 particle is described by the Euclidean action

$$
\int_{\mathrm{AdS}} d^{d+1} x \sqrt{g}\left[\frac{1}{2}\left(D_{\mu} A_{\nu}\right)^{2}-\frac{1}{2}\left(D^{\mu} A_{\mu}\right)^{2}+\frac{1}{2} M^{2} A^{\mu} A_{\mu}-A_{\mu} j^{\mu}\right],
$$

\footnotetext{
${ }^{1}$ On the submanifold $X^{2}+1=W^{2}=X \cdot W=0$, the order of the operators $K_{A}$ and $\nabla^{A}$ is not important because $\nabla \cdot K=K \cdot \nabla$.
} 
where $D_{\mu}$ is the AdS covariant derivative and $j^{\mu}$ is a classical source. This action gives rise to the Proca equation

$$
D^{2} A_{\mu}-D_{\mu}\left(D^{\nu} A_{\nu}\right)-M^{2} A_{\mu}=-j_{\mu} .
$$

Taking the divergence of this equation in the absence of source, we conclude that $D^{\nu} A_{\nu}=0$. Thus, in the absence of source, the Proca equation is equivalent to $D^{2} A_{\mu}=M^{2} A_{\mu}$ and $D^{\nu} A_{\nu}=0$.

A massive spin 2 particle is described by the Euclidean action [18, 31, 32]

$$
\begin{aligned}
\int_{\mathrm{AdS}} d^{d+1} x \sqrt{g}[ & \frac{1}{2}\left(D_{\mu} h_{\nu \alpha}\right)^{2}-\frac{1}{2}\left(D_{\mu} h\right)^{2}+D^{\mu} h_{\mu \nu} D^{\nu} h-D_{\mu} h_{\nu \alpha} D^{\alpha} h^{\nu \mu} \\
& \left.+d\left(h_{\mu \nu}\right)^{2}+\frac{d}{2} h^{2}+\frac{1}{2}\left(M^{2}+2\right)\left(h_{\mu \nu}^{2}-h^{2}\right)-T^{\mu \nu} h_{\mu \nu}\right],
\end{aligned}
$$

where $T^{\mu \nu}$ is a classical source and $h=g^{\mu \nu} h_{\mu \nu}$ is the trace of the field $h_{\mu \nu}$. This action can be obtained from the Einstein-Hilbert action for the metric $g_{\mu \nu}+h_{\mu \nu}$ in the presence of a negative cosmological constant equal to $-d(d-1) / 2$, by expanding to quadratic order in the metric fluctuation $h_{\mu \nu}$, and adding the Fierz-Pauli mass term $\frac{1}{2}\left(M^{2}+2\right)\left(h_{\mu \nu}^{2}-h^{2}\right)$ to the lagrangian. The equation of motion can be written as [18]

$$
\begin{aligned}
& \left(D^{2}-M^{2}\right) h_{\mu \nu}-D_{\mu} D^{\sigma} h_{\sigma \nu}-D_{\nu} D^{\sigma} h_{\mu \sigma}-2 g_{\mu \nu} h \\
& +\left(D_{\mu} D_{\nu}-\frac{M^{2}+2}{d-1} g_{\mu \nu}\right) h=-T_{\mu \nu}+\frac{1}{d-1} g_{\mu \nu} T_{\sigma}{ }^{\sigma},
\end{aligned}
$$

where we recall that the AdS radius is 1 in our units. By taking the trace and the divergence of this equation in the absence of source, we can derive

$$
D^{2} h_{\mu \nu}=M^{2} h_{\mu \nu}, \quad D^{\mu} h_{\mu \nu}=0, \quad h_{\mu}{ }^{\mu}=0 .
$$

The generalization of these equations to higher spin is more involved. Starting from spin 3 one must either introduce non-local terms or auxiliary fields [33-35]. However, on-shell, these equations always reduce to

$$
D^{2} h_{\mu_{1} \ldots \mu_{J}}=M^{2} h_{\mu_{1} \ldots \mu_{J}}, \quad D^{\mu} h_{\mu \mu_{2} \ldots \mu_{J}}=0, \quad h^{\mu}{ }_{\mu \mu_{3} \ldots \mu_{J}}=0 .
$$

This will be enough for our purposes, since it determines all poles associated with propagating degrees of freedom.

\subsection{Bulk-to-bulk propagator}

To construct the bulk-to-bulk propagator of a spin $J$ field between points $X_{1}$ and $X_{2}$, respectively with polarization vectors $W_{1}$ and $W_{2}$, we need to consider polynomials of degree $J$ in both $W_{1}$ and $W_{2}$ that can be constructed from the three possible scalar products $W_{1} \cdot W_{2}, X_{1} \cdot W_{2}$ and $X_{2} \cdot W_{1}$. The coefficient of each term can be a generic function of the chordal distance $u=-1-X_{1} \cdot X_{2}$. Thus we write with full generality

$$
\Pi_{\Delta, J}\left(X_{1}, X_{2}, W_{1}, W_{2}\right)=\sum_{k=0}^{J}\left(W_{12}\right)^{J-k}\left(\left(W_{1} \cdot X_{2}\right)\left(W_{2} \cdot X_{1}\right)\right)^{k} g_{k}(u),
$$

where we introduced the notation $W_{12}=W_{1} \cdot W_{2}$. 
To see how this formalism relates to the more conventional treatment, let us consider the simple case of $J=1$ and arbitrary dimension $\Delta$. In this case we have

$$
\Pi\left(X_{1}, X_{2}, W_{1}, W_{2}\right)=W_{12} g_{0}(u)+\left(W_{1} \cdot X_{2}\right)\left(W_{2} \cdot X_{1}\right) g_{1}(u) .
$$

Next we should act with the projector operator (2.12) to recover the components of the propagator as an embedding tensor

$$
\begin{aligned}
\Pi_{A, B}(X, Y)= & \left(\eta_{A B}+X_{A} X_{B}+Y_{A} Y_{B}-(1+u) X_{A} Y_{B}\right) g_{0}(u) \\
& +\left(X_{B}-(1+u) Y_{B}\right)\left(Y_{A}-(1+u) X_{A}\right) g_{1}(u)
\end{aligned}
$$

Finally we can project to some AdS coordinate system using (2.8). The terms proportional to $X_{A}$ or $Y_{B}$ are then seen to have a vanishing projection. The result can be expressed, in terms of the usual tensor structures constructed from derivatives of the chordal distance between both points, as

$$
\Pi_{\mu, \nu}(x, y)=-\frac{\partial^{2} u}{\partial x^{\mu} \partial y^{\nu}} g_{0}(u)+\frac{\partial u}{\partial x^{\mu}} \frac{\partial u}{\partial y^{\nu}} g_{1}(u) .
$$

Let us return to the problem of finding the general form of the functions $g_{k}(u)$ in (3.49). Based on a similar analysis in flat space that we include in appendix A, there is an alternative way other than (3.7) of writing the propagator that turns out to simplify the computation,

$$
\Pi_{\Delta, J}\left(X_{1}, X_{2}, W_{1}, W_{2}\right)=\sum_{k=0}^{J}\left(W_{12}\right)^{J-k}\left(\left(W_{1} \cdot \nabla_{1}\right)\left(W_{2} \cdot \nabla_{2}\right)\right)^{k} f_{k}(u) .
$$

The equivalence of expressions (3.7) and (3.11) relates the functions $g_{k}(u)$ and $f_{k}(u)$ through

$$
g_{k}(u)=\sum_{i=k}^{J}(-1)^{i+k}\left(\frac{i !}{k !}\right)^{2} \frac{1}{(i-k) !} f_{i}^{(i+k)}(u),
$$

where $f_{i}^{(k)}(u)=\partial_{u}^{k} f_{i}(u)$ denotes the $k$-th derivative of $f_{i}(u)$.

The equations for the bulk-to-bulk propagator of a massive spin $J$ field are given by

$$
\begin{aligned}
\left(\nabla_{1}^{2}-\Delta(\Delta-d)+J\right) \Pi_{\Delta, J}\left(X_{1}, X_{2}, W_{1}, W_{2}\right) & =-\delta\left(X_{1}, X_{2}\right)\left(W_{12}\right)^{J}+\ldots \\
\nabla_{1} \cdot K_{1} \Pi_{\Delta, J}\left(X_{1}, X_{2}, W_{1}, W_{2}\right) & =\ldots
\end{aligned}
$$

where we wrote the mass squared in (3.6) in AdS units as $M^{2}=\Delta(\Delta-d)-J$, such that $\Delta$ is the dimension of the dual operator. In these equations, the dots represent local source terms that are not important for the propagating degrees of freedom. As we shall see, they only change the propagator by contact terms. Since we will reproduce known formulae for lower spin fields, and also to make explicit our normalisation of the delta function singularity in the propagator equation, it is helpful to write these two equations in terms 
of components of the physical tensors. A mechanical computation shows that acting with the projector (2.12) we obtain the familiar equations

$$
\begin{aligned}
\left(D_{1}^{2}-\Delta(\Delta-d)+J\right) \Pi_{\mu_{1} \ldots \mu_{J}, \nu_{1} \ldots \nu_{J}}\left(x_{1}, x_{2}\right) & =-g_{\mu_{1}\left\{\nu_{1}\right.} \cdots g_{\left.\left|\mu_{J}\right| \nu_{J}\right\}} \delta\left(x_{1}, x_{2}\right)+\ldots, \\
D_{1}^{\mu_{1}} \Pi_{\mu_{1} \ldots \mu_{J}, \nu_{1} \ldots \nu_{J}}\left(x_{1}, x_{2}\right) & =\ldots
\end{aligned}
$$

where $D_{1}$ is the covariant derivative acting on functions of $x_{1}$ and we use the same convention for index symmetrization as given in (2.15).

The simplicity brought by the formalism can now be appreciated by the action of the Laplacian on our ansatz (3.11). One obtains for the propagator equation (3.13) the expression

$$
\begin{aligned}
& \left(W_{12}\right)^{J} \delta\left(X_{1}, X_{2}\right)+\cdots=\sum_{k=0}^{J}\left(W_{12}\right)^{J-k-1}\left[2(J-k)\left(W_{1} \cdot \nabla_{1}\right)^{k+1}\left(W_{2} \cdot \nabla_{2}\right)^{k}\left(X_{1} \cdot W_{2}\right)\right. \\
& \left.+W_{12}\left(\left(W_{1} \cdot \nabla_{1}\right)\left(W_{2} \cdot \nabla_{2}\right)\right)^{k}\left(u(2+u) \partial_{u}^{2}+(d+1)(1+u) \partial_{u}+k(2+k-2 J)-\Delta(\Delta-d)\right)\right] f_{k} .
\end{aligned}
$$

Although it is not explicit, this equation is actually symmetric under exchange of points 1 and 2. In fact, the term $\left(X_{1} \cdot W_{2}\right) f_{k}(u)$, arising from the first line, can be written as

$$
-W_{2} \cdot \nabla_{2} \int^{u} d u^{\prime} f_{k}\left(u^{\prime}\right)
$$

so the tensor structure of this term can be obtain from that of the second line in (3.17) by setting $k \rightarrow k+1$. Further simplification is achieved by using instead the $k$-th derivative of $f_{k}$, since in (3.12) there are always at least $k$ derivatives of $f_{k}$. Thus, using the shorthand notation $h_{k}=f_{k}^{(k)},(3.17)$ becomes

$$
\begin{gathered}
\left(u(2+u) \partial_{u}^{2}+(d+1)(1+u) \partial_{u}-\Delta(\Delta-d)\right) h_{0}=0, \\
\left(u(2+u) \partial_{u}^{2}+(d+1+2 k)(1+u) \partial_{u}+2 k(k-J+1)-\Delta(\Delta-d)\right) h_{k}=2(J+1-k) h_{k-1},
\end{gathered}
$$

for $k=0$ and $k>0$, respectively. The former is nothing more than the equation for the scalar propagator. Solving the latter for $J$ up to 7 we found a recurrence relation for $h_{k}$ in terms of $h_{k-1}$ and $h_{k-2}$. Inspired by this result we conjecture that the general solution is defined recursively by

$$
h_{k}=c_{k}\left((d-2 k+2 J-1)\left((d+J-2) h_{k-1}+(1+u) h_{k-1}^{\prime}\right)+(2-k+J) h_{k-2}\right),
$$

where

$$
c_{k}=-\frac{(1+J-k)}{k(d+2 J-k-2)(\Delta+J-k-1)(d-\Delta+J-k-1)},
$$

and

$$
h_{0}(u)=\frac{\Gamma(\Delta)}{2 \pi^{\frac{d}{2}} \Gamma\left(\Delta+1-\frac{d}{2}\right)}(2 u)^{-\Delta}{ }_{2} F_{1}\left(\Delta, \Delta+\frac{1-d}{2}, 2 \Delta-d+1,-\frac{2}{u}\right) .
$$

The normalization of $h_{0}$ is fixed by the $\delta$-function source in the propagator equation. The equation for the divergence (3.14) was checked to hold for $J$ up to 6 . Previous results for propagators in AdS were confirmed for $J=1$ and $J=2$, as we now discuss [18, 21, 22, 36]. 


\subsubsection{Spin 1}

In the case $J=1$ explicitly considered above, using (3.12) and $f_{i}^{(i+k)}=h_{i}^{(k)}$, it is simple to see that the functions of the chordal distance that multiply the different tensor structures, as described by (3.10), are given by

$$
\begin{aligned}
& g_{0}(u)=(d-\Delta) F_{1}(u)-\frac{1+u}{u} F_{2}(u), \\
& g_{1}(u)=\frac{(1+u)(d-\Delta)}{u(2+u)} F_{1}(u)-\frac{d+(1+u)^{2}}{u^{2}(2+u)} F_{2}(u),
\end{aligned}
$$

where

$$
\begin{aligned}
& F_{1}(u)=\mathcal{N}(2 u)^{-\Delta}{ }_{2} F_{1}\left(\Delta, \frac{1-d+2 \Delta}{2}, 1-d+2 \Delta,-\frac{2}{u}\right), \\
& F_{2}(u)=\mathcal{N}(2 u)^{-\Delta}{ }_{2} F_{1}\left(\Delta+1, \frac{1-d+2 \Delta}{2}, 1-d+2 \Delta,-\frac{2}{u}\right),
\end{aligned}
$$

with

$$
\mathcal{N}=\frac{\Gamma(\Delta+1)}{2 \pi^{d / 2}(d-1-\Delta)(\Delta-1) \Gamma\left(\Delta+1-\frac{d}{2}\right)}
$$

\subsubsection{Spin 2}

To make contact with previous results in the literature we will compare (3.7) for $J=2$ with the result for massive symmetric spin 2 field in [18]. The solution of the equations of motion for a symmetric spin two propagator can be organized in five structures (including the trace part for now)

$$
G_{\mu_{1} \mu_{2} ; \nu_{1} \nu_{2}}(u)=\sum_{i=1}^{5} A^{(i)}(u) T_{\mu_{1} \mu_{2} ; \nu_{1} \nu_{2}}^{(i)}
$$

where $T_{\mu_{1} \mu_{2} ; \nu_{1} \nu_{2}}^{(i)}$ are the five independent structures

$$
\begin{aligned}
& T_{\mu_{1} \mu_{2} ; \nu_{1} \nu_{2}}^{(1)}=g^{\mu_{1} \mu_{2}} g^{\nu_{1} \nu_{2}}, \\
& T_{\mu_{1} \mu_{2} ; \nu_{1} \nu_{2}}^{(2)}=\partial_{\mu_{1}} u \partial_{\mu_{2}} u \partial_{\nu_{1}} u \partial_{\nu_{2}} u, \\
& T_{\mu_{1} \mu_{2} ; \nu_{1} \nu_{2}}^{(3)}=\partial_{\mu_{1}} \partial_{\nu_{1}} u \partial_{\mu_{2}} \partial_{\nu_{2}} u+\partial_{\mu_{1}} \partial_{\nu_{2}} u \partial_{\mu_{2}} \partial_{\nu_{1}} u, \\
& T_{\mu_{1} \mu_{2} ; \nu_{1} \nu_{2}}^{(4)}=\partial_{\nu_{1}} u \partial_{\nu_{2}} u g^{\mu_{1} \mu_{2}}+\partial_{\mu_{1}} u \partial_{\mu_{2}} u g^{\nu_{1} \nu_{2}}, \\
& T_{\mu_{1} \mu_{2} ; \nu_{1} \nu_{2}}^{(5)}=\partial_{\mu_{1}} \partial_{\nu_{1}} u \partial_{\mu_{2}} u \partial_{\nu_{2}} u+\partial_{\mu_{2}} \partial_{\nu_{1}} u \partial_{\mu_{1}} u \partial_{\nu_{2}} u+\left(\nu_{1} \leftrightarrow \nu_{2}\right),
\end{aligned}
$$

and the specific form of the functions $A^{(i)}(u)$ is given in [18]. However, there are only three symmetric and traceless structures that can be constructed from $T_{\mu_{1} \mu_{2} ; \nu_{1} \nu_{2}}^{(i)}$. These 
correspond to following structures in the embedding formalism

$$
\begin{aligned}
& \left(W_{12}\right)^{2} \rightarrow \frac{T_{\mu_{1} \mu_{2} ; \nu_{1} \nu_{2}}^{(3)}}{2}-\frac{T_{\mu_{1} \mu_{2} ; \nu_{1} \nu_{2}}^{(1)}(1+d-u(2+u))}{(1+d)^{2}}-\frac{T_{\mu_{1} \mu_{2} ; \nu_{1} \nu_{2}}^{(4)}}{1+d}
\end{aligned}
$$

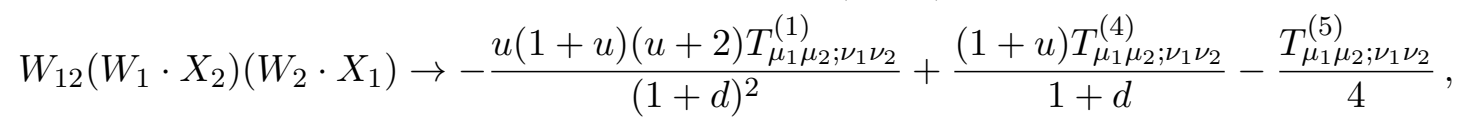

$$
\begin{aligned}
& \left(W_{1} \cdot X_{2}\right)\left(W_{2} \cdot X_{1}\right)^{2} \rightarrow \frac{u^{2}(2+u)^{2} T_{\mu_{1} \mu_{2} ; \nu_{1} \nu_{2}}^{(1)}}{(1+d)^{2}}+T_{\mu_{1} \mu_{2} ; \nu_{1} \nu_{2}}^{(2)}-\frac{u(u+2) T_{\mu_{1} \mu_{2} ; \nu_{1} \nu_{2}}^{(4)}}{1+d} .
\end{aligned}
$$

Therefore, the functions in the expansion (3.27) are given by

$$
\begin{aligned}
& A^{(2)}(u)=g_{2}(u), \quad A^{(3)}(u)=\frac{1}{2} g_{0}(u), \quad A^{(5)}(u)=-\frac{1}{4} g_{1}(u), \\
& A^{(1)}(u)=-\frac{1+d-u(2+u)}{(1+d)^{2}} g_{0}(u)-\frac{u(1+u)(u+2)}{(1+d)^{2}} g_{1}(u)+\frac{u^{2}(2+u)^{2}}{(1+d)^{2}} g_{2}(u), \\
& A^{(4)}(u)=-\frac{1}{1+d} g_{0}(u)+\frac{(1+u)}{1+d} g_{1}(u)-\frac{u(u+2)}{1+d} g_{2}(u) .
\end{aligned}
$$

Using the results of the previous section for $J=2$, we recover the results of [18]. This is the full result for the propagator up to contact terms. In section 4.4 we shall discuss in detail the contact terms for the spin 2 case.

\subsection{Bulk-to-boundary propagator}

In the embedding formalism, the bulk-to-boudary propagator of a spin $J$ and dimension $\Delta$ field has the simple form

$$
\Pi_{\Delta, J}(X, P ; W, Z)=\mathcal{C}_{\Delta, J} \frac{((-2 P \cdot X)(W \cdot Z)+2(W \cdot P)(Z \cdot X))^{J}}{(-2 P \cdot X)^{\Delta+J}} .
$$

This is the unique structure compatible with conformal symmetry, which in this formalism is encoded by the constraint

$$
\Pi_{\Delta, J}\left(X, \lambda P ; \alpha_{1} W, \alpha_{2} Z+\beta P\right)=\lambda^{-\Delta}\left(\alpha_{1} \alpha_{2}\right)^{J} \Pi_{\Delta, J}(X, P ; W, Z),
$$

for arbitrary constants $\lambda, \alpha_{1}, \alpha_{2}$ and $\beta$. The normalization constant $\mathcal{C}_{\Delta, J}$ is fixed by considering the bulk-to-bulk propagator, properly normalised by its short distance behaviour, and then sending one of the bulk points to the boundary, according to

$$
\lim _{\lambda \rightarrow \infty} \lambda^{\Delta} \Pi_{\Delta, J}\left(X, \lambda P+O\left(\lambda^{-1}\right) ; W, Z\right)=\Pi_{\Delta, J}(X, P ; W, Z) .
$$

Let us check that this works for the bulk-to-bulk propagator computed in the previous section. First we observe that the recurrence relation (3.20) is simplified in the limit $u \rightarrow \infty$. In this limit, this relation preserves the same asymptotic behaviour for all functions $h_{k}(u)$. Hence, from the asymptotic behaviour of $h_{0}(u)$, we conclude that $h_{k} \approx s_{k} u^{-\Delta}$, and therefore (3.20) gives rise to a recursion relation for $s_{k}$,

$$
s_{k}=c_{k}\left((d-2 k+2 J-1)(d-\Delta+J-2) s_{k-1}+(2-k+J) s_{k-2}\right) .
$$


This equation has the following solution

$$
s_{k}=s_{0} \frac{J !}{k !(J-k) !} \frac{(-1)^{k}}{(J+\Delta-k-1)_{k}},
$$

which implies after the use of (3.12) that

$$
g_{k}(u) \approx s_{0} \frac{J !}{k !(J-k) !} \frac{J+\Delta-1}{\Delta-1} u^{-\Delta-k} .
$$

It is then clear that we recover the form of the bulk-to-boudary propagator (3.39),

$$
\Pi_{\Delta, J}(X, P ; W, Z)=s_{0} 2^{\Delta} \frac{(\Delta-1+J)}{\Delta-1} \frac{(2(W \cdot P)(X \cdot Z)-2(W \cdot Z)(X \cdot P))^{J}}{(-2 X \cdot P)^{J+\Delta}} .
$$

The constant $s_{0}$ is fixed by the normalisation imposed by the delta function source in the propagator equation. We can just fix it by looking at the asymptotic behaviour of the function $h_{0}(u)$, which fixes the normalisation constant $\mathcal{C}_{\Delta, J}$ introduced in (3.39) to be

$$
\mathcal{C}_{\Delta, J}=\frac{(J+\Delta-1) \Gamma(\Delta)}{2 \pi^{d / 2}(\Delta-1) \Gamma(\Delta+1-h)} .
$$

\subsection{Short distance limit}

Next we consider the short distance limit where $u \rightarrow 0$. Our goal is to check computations done in [19] that can also be done by directly computing the difference between the short distance behaviour of spin $J$ propagators of dimension $\Delta$ and $d-\Delta$. First, let us note that our solution for the spin $J$ propagator is based on the recursion relation (3.19), where the seed is given by the scalar propagator (3.22). It so happens that the scalar propagator diverges at short distances. However, the coefficients of all the divergent terms are invariant under $\Delta \rightarrow d-\Delta$. Therefore, since the recurrence relation is also invariant under this transformation, the difference of the spin $J$ propagators of dimension $\Delta$ and $d-\Delta$ is finite in the limit $u \rightarrow 0$. Defining $\widetilde{h}_{0}$ as the difference of $h_{0}$ for dimension $\Delta$ and $d-\Delta$ we can obtain, from the explicit result (3.22),

$$
\widetilde{h}_{0}(u)=\sin \left(\frac{\pi}{2}(d-2 \Delta)\right) \sum_{k=0}^{\infty} \frac{\Gamma(d-\Delta+k) \Gamma(\Delta+k)}{\pi^{\frac{d+1}{2}} 2^{d+k} k ! \Gamma\left(\frac{1+d}{2}+k\right)}(-u)^{k} .
$$

To make contact with the computation of [19] we only need to consider the difference of the trace of the spin $J$ propagators. In the embedding formalism the trace can be obtained simply by acting on the propagator with the operator

$$
\frac{1}{\left(J !\left(\frac{d-1}{2}\right)_{J}\right)^{2}}\left(K_{1} \cdot K_{2}\right)^{J},
$$

where $K$ is defined in (2.12) and we were careful with the numerical factor to obtain exactly the trace. We show in appendix B that in the limit $u \rightarrow 0$ the action of this operator on the difference of propagators $\widetilde{\Pi}$ is

$$
\left(K_{1} \cdot K_{2}\right)^{J} \widetilde{\Pi}_{\Delta, J}\left(X_{1}, X_{2}, W_{1}, W_{2}\right) \approx\left(K_{1} \cdot K_{2}\right)^{J}\left(W_{12}\right)^{J} \widetilde{g}_{0}(u=0) .
$$


To compute $\widetilde{g}_{0}(u=0)$ we need to use the relation (3.12) involving a sum over all the $\widetilde{h}_{k}(u=0)$, which in turn can be determined using the recursion relation (3.20) and the series expansion (3.47) of $\widetilde{h}_{0}$. We did this computation up to $J=12$ and verified that the result for the difference of the trace of the propagators exactly matches that of [19],

$$
g(J) \frac{(\Delta+J-1)(\Delta-J-d+1) \Gamma(\Delta-1) \Gamma(d-1-\Delta) \sin \left(\frac{\pi}{2}(d-2 \Delta)\right)}{2^{d} \pi^{\frac{d+1}{2}} \Gamma\left(\frac{1+d}{2}\right)},
$$

where $g(J)$ is given by

$$
\begin{array}{ll}
g(J)=\frac{(2 J+d-2)(J+d-3) !}{(d-2) ! J !}, & d \geq 3, \\
g(0)=1, \quad g(J)=2, & d=2 .
\end{array}
$$

\subsection{Massless limit}

To analyse the massless limit of the spin $J$ bulk-to-bulk propagator let us introduce the new representation

$$
\Pi_{\Delta, J}=\left(W_{12}\right)^{J} G(u)+W_{1} \cdot \nabla\left(\sum_{k=1}^{J}\left(W_{12}\right)^{J-k}\left(W_{2} \cdot X_{1}\right)^{k}\left(W_{1} \cdot X_{2}\right)^{k-1} L_{k}(u)\right) .
$$

Comparing with expression (3.7) we conclude that

$$
\begin{aligned}
& g_{0}(u)=G(u)+L_{1}(u), \\
& g_{k}(u)=-L_{k}^{\prime}(u)+(k+1) L_{k+1}(u), \quad k=1, \ldots, J-1, \\
& g_{J}(u)=-L_{J}^{\prime}(u) .
\end{aligned}
$$

These relations can be inverted to give the functions $L_{k}$ in terms of the functions $g_{k}$,

$$
L_{k}(u)=-\sum_{l=k}^{J} \frac{\Gamma(l+1)}{\Gamma(k+1)} \underbrace{\int^{u} \ldots \int d u^{\prime}}_{l-k+1} g_{l}\left(u^{\prime}\right) .
$$

The function $G(u)$ follows after a simple manipulation,

$$
\begin{aligned}
G(u) & =g_{0}(u)+\sum_{l=1}^{J} \Gamma(l+1) \underbrace{\int^{u} \ldots \int d u^{\prime}}_{l} g_{l}\left(u^{\prime}\right), \\
& =\sum_{l=0}^{J} \sum_{i=l}^{J}(-1)^{i+l} \frac{\Gamma^{2}(i+1)}{\Gamma(l+1) \Gamma(i-l+1)} h_{i}(u)=h_{0}(u),
\end{aligned}
$$

where we used equation $(3.12)$ and $f_{i}^{(i+k)}(u)=h_{i}^{(k)}(u)$.

Expression (3.53) for the bulk-to-bulk propagator is very convenient to study the massless limit. In this case, gauge invariance implies that the propagator is always coupled to conserved currents. If the current is also traceless, then the functions $L_{k}$ in (3.53) do not 
contribute to physical processes (because their contribution vanishes after integrating by parts). In other words, the function $G(u)$ is the only physical degree of freedom. This is the same result found in [37] for every spin $J$. Notice that, one must be careful in intermediate calculations because the gauge artifacts $L_{k}(u)$ diverge in the massless limit $\Delta \rightarrow J+d-2$ (this is clear from the explicit form of the coefficient $a_{k}$ in (3.21)). In general, however, the conserved current is not traceless. The analysis of this case is more involved and was considered in [38]. The main result is that the structures that couple to the multiple traces of the current also remain finite in the massless limit. To clarify this point we now review the spin 2 case.

\subsubsection{Graviton}

As explained above, it is important to isolate physical components from gauge artifacts. The massive spin 2 symmetric and traceless propagator (3.27) can be rewritten in the form,

$$
\begin{aligned}
G_{\mu_{1} \mu_{2} ; \nu_{1} \nu_{2}}= & \frac{\partial_{\mu_{1}} \partial_{\nu_{1}} u \partial_{\mu_{2}} \partial_{\nu_{2}} u+\partial_{\mu_{1}} \partial_{\nu_{2}} u \partial_{\mu_{2}} \partial_{\nu_{1}} u}{2} G(u)+g_{\mu_{1} \mu_{2}} g_{\nu_{1} \nu_{2}} H(u) \\
& +\partial_{\left(\mu_{1}\right.}\left[\partial_{\left.\mu_{2}\right)} \partial_{\nu_{1}} u \partial_{\nu_{2}} u X(u)\right]+\partial_{\left(\nu_{1}\right.}\left[\partial_{\left.\nu_{2}\right)} \partial_{\mu_{1}} u \partial_{\mu_{2}} u X(u)\right] \\
& +\partial_{\left(\mu_{1}\right.}\left[\partial_{\left.\mu_{2}\right)} u \partial_{\nu_{1}} u \partial_{\nu_{2}} u Y(u)\right]+\partial_{\left(\nu_{1}\right.}\left[\partial_{\left.\nu_{2}\right)} u \partial_{\mu_{1}} u \partial_{\mu_{2}} u Y(u)\right] \\
& +\partial_{\mu_{1}}\left[\partial_{\mu_{2}} u Z(u)\right] g_{\nu_{1} \nu_{2}}+\partial_{\nu_{1}}\left[\partial_{\nu_{2}} u Z(u)\right] g_{\mu_{1} \mu_{2}},
\end{aligned}
$$

where $($,$) denotes symmetrization. Only the first line in this expression gives a finite$ contribution when coupled to a conserved symmetric tensor (not necessarily traceless). The physical components $G(u)$ and $H(u)$ can be written in terms of $h_{0}(u)$ as

$$
\begin{aligned}
G(u)= & h_{0}(u) \\
H(u)= & -\frac{1}{d(d-1+\Delta(\Delta-d))}\left(d(2 d-4+\Delta(\Delta-d)) \int_{u}^{\infty} d u^{\prime} \int_{u^{\prime}}^{\infty} d u^{\prime \prime} h_{0}\left(u^{\prime \prime}\right)\right. \\
& \left.-d(1+u) \int_{u}^{\infty} d u^{\prime} h_{0}\left(u^{\prime}\right)+(d+\Delta(\Delta-d)) h_{0}\right)
\end{aligned}
$$

Both functions are regular in the massless limit and agree with [20].

\section{Split representation of AdS propagators}

There is an alternative representation for bulk-to-bulk propagators which is often termed as split representation. The aim of this section is to introduce this representation for spin $J$ fields and explicitly compute the propagator in some examples. We start by defining a basis of spin $J$ harmonic functions, denoted as $\Omega_{\nu, J}$. As will be shown, the propagator can be written as a linear combination of these functions.

\subsection{Spin $J$ harmonic functions in AdS}

The integral over the boundary point of the product of two bulk-to-boudary propagators, with dimensions $h+i \nu$ and $h-i \nu$, is by construction invariant under the exchange $\nu \leftrightarrow-\nu$. Moreover, it depends just on the bulk points $X_{1}$ and $X_{2}$ and polarization vectors $W_{1}$ and 


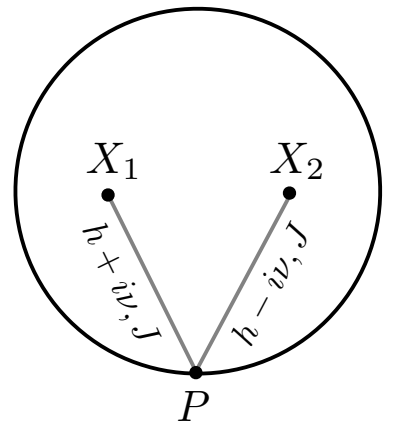

Figure 2. Representation of AdS harmonic function $\Omega_{\nu, J}$ in terms of two spin $J$ bulk-to-boundary propagators of dimension $h \pm i \nu$ integrated over the boundary point.

$W_{2}$. This is schematically represented in figure 2 and leads to the following definition of the AdS harmonic function

$$
\Omega_{\nu, J}\left(X_{1}, X_{2} ; W_{1}, W_{2}\right)=\frac{\nu^{2}}{\pi J !(h-1)_{J}} \int_{\partial} d P \Pi_{h+i \nu, J}\left(X_{1}, P ; W_{1}, D_{Z}\right) \Pi_{h-i \nu, J}\left(X_{2}, P ; W_{2}, Z\right),
$$

where $Z$ denotes a polarization vector on the boundary and $h=d / 2$ was introduced for convenience. The operator $D_{Z}$, given by,

$$
D_{Z}^{A}=\left(h-1+Z \cdot \frac{\partial}{\partial Z}\right) \frac{\partial}{\partial Z_{A}}-\frac{1}{2} Z^{A} \frac{\partial^{2}}{\partial Z \cdot \partial Z},
$$

is the boundary counterpart of (2.12) and implements index contraction of tensors defined on the boundary of AdS. In appendix C, we compute the integral (4.1) and show that it is given by a linear combination of two bulk-to-bulk propagators [21]

$$
\Omega_{\nu, J}\left(X_{1}, X_{2} ; W_{1}, W_{2}\right)=\frac{i \nu}{2 \pi}\left(\Pi_{h+i \nu, J}\left(X_{1}, X_{2} ; W_{1}, W_{2}\right)-\Pi_{h-i \nu, J}\left(X_{1}, X_{2} ; W_{1}, W_{2}\right)\right) .
$$

Notice that $\Omega_{\nu, J}$ is an eigenfunction of the Laplacian operator and is divergence free,

$$
\begin{aligned}
\left(\nabla_{1}^{2}+h^{2}+\nu^{2}+J\right) \Omega_{\nu, J}\left(X_{1}, X_{2} ; W_{1}, W_{2}\right) & =0 \\
\nabla_{1} \cdot K_{1} \Omega_{\nu, J}\left(X_{1}, X_{2} ; W_{1}, W_{2}\right) & =0 .
\end{aligned}
$$

These properties follow from (4.3) and equations (3.13)-(3.14) for the bulk-to-bulk propagator.

Besides being an eigenfunction of the Laplacian, $\Omega_{\nu, J}$ satisfies an orthogonality relation. To see this, consider the integral

$$
\frac{1}{J !\left(\frac{d-1}{2}\right)_{J}} \int_{\mathrm{AdS}} d Y \Omega_{\bar{\nu}, J}\left(X_{1}, Y ; W_{1}, K\right) \Omega_{\nu, J}\left(Y, X_{2} ; W, W_{2}\right)=C_{\nu, \bar{\nu}}\left(X_{1}, X_{2} ; W_{1}, W_{2}\right),
$$

This object can only depend on the invariants $X_{1} \cdot X_{2}, W_{1} \cdot W_{2}$ and $\left(W_{1} \cdot X_{2}\right)\left(W_{2} \cdot X_{1}\right)$. Therefore, it is invariant under the exchange $\left(X_{1}, W_{1}\right) \leftrightarrow\left(X_{2}, W_{2}\right)$. By construction, $C_{\nu, \bar{\nu}}$ is an eigenfunction of the Laplacian. Thus, the expression

$$
\left(\nabla_{1}^{2}-\nabla_{2}^{2}\right) C_{\nu, \bar{\nu}}\left(X_{1}, X_{2} ; W_{1}, W_{2}\right)=\left(\nu^{2}-\bar{\nu}^{2}\right) C_{\nu, \bar{\nu}}\left(X_{1}, X_{2} ; W_{1}, W_{2}\right)=0,
$$


must vanish because it must be both antisymmetric and symmetric under the permutation $\left(X_{1}, W_{1}\right) \leftrightarrow\left(X_{2}, W_{2}\right)$. This means $C_{\nu, \bar{\nu}}$ only has support at $\bar{\nu}= \pm \nu$. Notice also that $C_{\nu, \bar{\nu}}$ is a harmonic function in the variables $X_{1}$ and $X_{2}$, so it should be proportional to $\Omega_{\nu, J}$. Thus

$$
C_{\nu, \bar{\nu}}\left(X_{1}, X_{2} ; W_{1}, W_{2}\right)=\frac{1}{2}[\delta(\nu+\bar{\nu})+\delta(\nu-\bar{\nu})] \Omega_{\nu, J}\left(X_{1}, X_{2} ; W_{1}, W_{2}\right),
$$

where the constant of proportionality was determined in appendix D. Integrating $C_{\nu, \bar{\nu}}$ over $\nu$, we find

$$
\frac{1}{J !\left(\frac{d-1}{2}\right)_{J}} \int_{\mathrm{AdS}} d Y \Omega_{\bar{\nu}, J}\left(X_{1}, Y ; W_{1}, K\right) \int d \nu \Omega_{\nu, J}\left(Y, X_{2} ; W, W_{2}\right)=\Omega_{\bar{\nu}, J}\left(X_{1}, X_{2} ; W_{1}, W_{2}\right),
$$

which implies that

$$
\int_{-\infty}^{\infty} d \nu \Omega_{\nu, J}\left(X_{1}, X_{2} ; W_{1}, W_{2}\right)=\delta\left(X_{1}, X_{2}\right)\left(W_{12}\right)^{J}+\left(W_{1} \cdot \nabla_{1}\right)\left(W_{2} \cdot \nabla_{2}\right) Q\left(X_{1}, X_{2} ; W_{1}, W_{2}\right)
$$

since we can always add to the right hand side of (4.9) a total derivative because $\Omega_{\bar{\nu}, J}$ has zero divergence. The function $Q$ can be written as

$$
Q\left(X_{1}, X_{2} ; W_{1}, W_{2}\right)=-\sum_{l=1}^{J} \int d \nu c_{J, l}(\nu)\left(\left(W_{1} \cdot \nabla_{1}\right)\left(W_{2} \cdot \nabla_{2}\right)\right)^{l-1} \Omega_{\nu, J-l}\left(X_{1}, X_{2} ; W_{1}, W_{2}\right) .
$$

This means that we can write the completeness relation ${ }^{2}$

$$
\sum_{l=0}^{J} \int d \nu c_{J, l}(\nu)\left(\left(W_{1} \cdot \nabla_{1}\right)\left(W_{2} \cdot \nabla_{2}\right)\right)^{l} \Omega_{\nu, J-l}\left(X_{1}, X_{2} ; W_{1}, W_{2}\right)=\delta\left(X_{1}, X_{2}\right)\left(W_{12}\right)^{J}
$$

where $c_{J, 0}(\nu)=1$. In appendix $\mathrm{D}$ we derive the general formula

$$
c_{J, l}(\nu)=\frac{2^{l}(J-l+1)_{l}\left(h+J-l-\frac{1}{2}\right)_{l}}{l !(2 h+2 J-2 l-1)_{l}(h+J-l-i \nu)_{l}(h+J-l+i \nu)_{l}},
$$

using a recursive argument to increase $J$ and $l$.

\subsection{Split representation}

Let us express the propagator as a linear combination of harmonic functions,

$$
\Pi_{\Delta, J}\left(X_{1}, X_{2} ; W_{1}, W_{2}\right)=\sum_{l=0}^{J} \int d \nu a_{l}(\nu)\left(\left(W_{1} \cdot \nabla_{1}\right)\left(W_{2} \cdot \nabla_{2}\right)\right)^{J-l} \Omega_{\nu, l}\left(X_{1}, X_{2} ; W_{1}, W_{2}\right),
$$

\footnotetext{
${ }^{2}$ We call this a completeness relation because if we use the representation (4.1) of the harmonic functions, equation (4.11) tells us that the functions $F_{\nu, l, P}(X, W) \equiv(W \cdot \nabla)^{l} \Pi_{h+i \nu, J-l}(X, W ; P, Z)$, with $\nu \in \mathbb{R}$, $P \in \mathbb{R}^{d}$ and $l=0,1, \ldots J$, form a complete basis for spin $J$ (symmetric and traceless) tensors in AdS.
} 


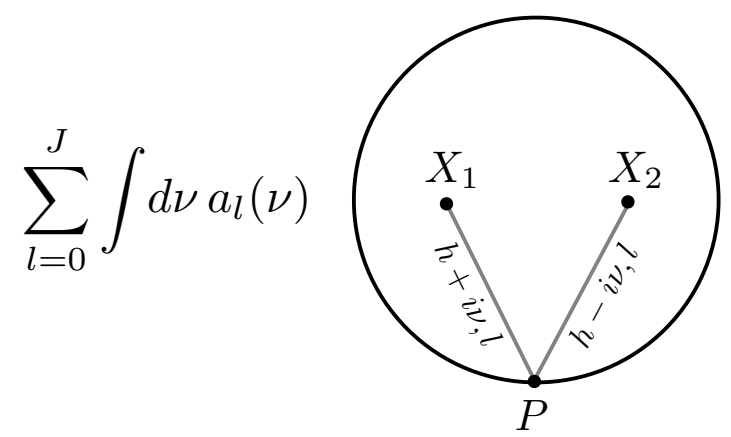

Figure 3. The split representation of the spin $J$ propagator obtained by integrating over $\nu$ and summing over the spin $l$ of two bulk-to-boundary propagators of dimension $h \pm i \nu$ integrated over the boundary point, according to (4.13).

as represented in figure 3 . This is the split representation of the propagator. The coefficients $a_{l}(\nu)$ of the split representation can be obtained using the equations of motion. More specifically, using the commutation relation

$$
\left[\nabla^{2},(W \cdot \nabla)^{n}\right]=-n\left(2 h-1+2 W \cdot \partial_{W}-n\right)(W \cdot \nabla)^{n},
$$

in equation (3.13), we find

$$
\begin{aligned}
& {\left[\Delta(\Delta-d)-J-\nabla_{1}^{2}\right] \Pi_{\Delta, J}\left(X_{1}, X_{2} ; W_{1}, W_{2}\right)=} \\
& \sum_{l=0}^{J} \int d \nu a_{l}(\nu)\left[(J-l)(2 h+J+l-2)+\nu^{2}+(\Delta-h)^{2}\right]\left(\left(W_{1} \cdot \nabla_{1}\right)\left(W_{2} \cdot \nabla_{2}\right)\right)^{J-l} \Omega_{\nu, l} .
\end{aligned}
$$

We shall assume that the traceless part of the contact terms has the following general form

$$
\begin{aligned}
& {\left[\Delta(\Delta-d)-J-\nabla_{1}^{2}\right] \Pi_{\Delta, J}\left(X_{1}, X_{2} ; W_{1}, W_{2}\right) } \\
= & \sum_{s=0}^{J} v_{J-s}\left(\left(W_{1} \cdot \nabla_{1}\right)\left(W_{2} \cdot \nabla_{2}\right)\right)^{J-s}\left[\left(W_{12}\right)^{s} \delta\left(X_{1}, X_{2}\right)\right],
\end{aligned}
$$

where $v_{J-s}$ are constants and $v_{0}=1$ is fixed by the normalisation of the delta-function term without derivatives. Using the representation (4.11) of the delta function, and comparing with (4.15) we obtain

$$
a_{l}(\nu)\left[(J-l)(2 h+J+l-2)+\nu^{2}+(\Delta-h)^{2}\right]=\sum_{s=l}^{J} v_{J-s} c_{s, s-l}(\nu),
$$

for $0 \leq l \leq J$. Since $c_{J, 0}=1$, the case $l=J$ gives immediately

$$
a_{J}(\nu)=\frac{1}{\nu^{2}+(\Delta-h)^{2}} .
$$

To determine the coefficients $a_{l}(\nu)$ for $l<J$ we look for a solution of (4.17) with the minimal number of poles in $\nu$. From the explicit form of $c_{s, s-l}(\nu)$ given in (4.12), we are 
led to the ansatz

$$
a_{l}(\nu)=\sum_{q=1}^{J-l} \frac{r_{l, q}}{\nu^{2}+(h+l+q-1)^{2}}, \quad l<J,
$$

where the $J$ and $\Delta$ dependence of the residue is implicit. To determine these residues we consider equation (4.17) when $\nu^{2} \rightarrow-(h+l+q-1)^{2}$. This gives

$$
\begin{aligned}
& r_{l, q}\left[(J-l)(2 h+J+l-2)-(h+l+q-1)^{2}+(\Delta-h)^{2}\right] \\
= & \sum_{s=l}^{J} v_{J-s} \lim _{\nu \rightarrow i(h+l+q-1)}\left[\nu^{2}+(h+l+q-1)^{2}\right] c_{s, s-l}(\nu) \\
= & \sum_{s=l+q}^{J} v_{J-s} \frac{(-1)^{q+1} 2^{s-l+1} s !(h+l+q-1)\left(h+l-\frac{1}{2}\right)_{s-l}}{(q-1) ! l !(s-l) !(s-l-q) !(2 h+2 l-1)_{s-l}(2 h+2 l+q-1)_{s-l}} .
\end{aligned}
$$

On the other hand, the limit $\nu^{2} \rightarrow \infty$ of (4.17) gives

$$
v_{J-l}=\sum_{q=1}^{J-l} r_{l, q} .
$$

Using these two equations, one finds that

$$
v_{k}=\frac{(-1)^{k} J !(2 h+2 J-2 k-2)_{k}}{2^{k} k !(J-k) !(h+J-k-1)_{k}(\Delta+J-k-1)_{k}(2 h-\Delta+J-k-1)_{k}},
$$

which in turn determines the residues $r_{l, q}$ through equation (4.20). As an example, we show the first coefficients,

$$
\begin{aligned}
& a_{J-1}(\nu)=-\frac{J}{y_{2}\left(\nu^{2}+(h+J-1)^{2}\right)}, \\
& a_{J-2}(\nu)=\frac{J(J-1)}{4 y_{3}(J+h-2)\left(\nu^{2}+(h+J-1)^{2}\right)}-\frac{J(J-1)}{4 y_{2}(J+h-2)\left(\nu^{2}+(h+J-2)^{2}\right)},
\end{aligned}
$$

with $y_{k}=(\Delta+J-k)(2 h-\Delta+J-k)$. The expression for other coefficients, $a_{J-k}(\nu)$, cannot be written explicitly in such a compact form. However, they are completely determined by $(4.20)$ and (4.22). In section 6 , we shall use a very different argument to derive a recursion relation that also fixes all coefficients $a_{l}(\nu)$.

In finding the solution for the coefficients $a_{l}(\nu)$ we made two simplifying assumptions: the structure of the contact terms in the propagator equation (4.16) and the existence of a minimal number of poles in $\nu$. In the next subsections, we consider the case of spin 1 and 2 and determine the full split representation. We will find that the above assumptions are indeed correct. 


\subsection{Spin 1}

The divergence of the spin 1 propagator vanishes at separate points. However, one must take special care with possible contact terms. Notice that, in general, these give non-zero contributions to Witten diagrams. To determine the possible contact terms we return to the Proca equation, which gives for the propagator

$$
\left(D_{1}^{2}+1-\Delta(\Delta-d)\right) \Pi_{\mu, \nu}\left(x_{1}, x_{2}\right)-D_{\mu} D^{\sigma} \Pi_{\sigma, \nu}\left(x_{1}, x_{2}\right)=-g_{\mu \nu} \delta\left(x_{1}, x_{2}\right) .
$$

In the embedding formalism this can be written as

$$
\left[\nabla_{1}^{2}+1-\Delta(\Delta-d)-\frac{2}{d-1}\left(W_{1} \cdot \nabla_{1}\right)\left(K_{1} \cdot \nabla_{1}\right)\right] \Pi_{\Delta, 1}\left(X_{1}, X_{2} ; W_{1}, W_{2}\right)=-W_{12} \delta\left(X_{1}, X_{2}\right) .
$$

It is straightforward to check that this equation is solved exactly by the split representation we found in the previous section,

$$
\begin{aligned}
\Pi_{\Delta, 1}\left(X_{1}, X_{2} ; W_{1}, W_{2}\right)= & \int \frac{d \nu \Omega_{\nu, 1}\left(X_{1}, X_{2} ; W_{1}, W_{2}\right)}{\nu^{2}+(\Delta-h)^{2}} \\
& -\int \frac{d \nu\left(W_{1} \cdot \nabla_{1}\right)\left(W_{2} \cdot \nabla_{2}\right) \Omega_{\nu, 0}\left(X_{1}, X_{2}\right)}{(\Delta-1)(2 h-\Delta-1)\left(\nu^{2}+h^{2}\right)} .
\end{aligned}
$$

In this calculation we used the basic properties (4.4) and (4.5) of the harmonic functions, the simple commutators (D.25) given in appendix D and the completeness relation (4.11). We conclude that, in this case, the coefficients $a_{1}(\nu)$ and $a_{0}(\nu)$ are entirely determined by their poles (4.18) and (4.23) without any additional regular piece.

\subsection{Spin 2}

The massive spin 2 propagator is traceless and divergenceless when the two bulk points it connects are different. To determine possible contact terms we write the full propagator as a sum of three terms. The first term is the traceless part that we discussed so far. Since it is traceless it can be written as a polynomial in $W_{1}$ and $W_{2}$,

$$
\Pi_{\Delta, 2}\left(X_{1}, X_{2} ; W_{1}, W_{2}\right)=\sum_{l=0}^{2} \int d \nu a_{l}(\nu)\left(\left(W_{1} \cdot \nabla_{1}\right)\left(W_{2} \cdot \nabla_{2}\right)\right)^{2-l} \Omega_{\nu, l}\left(X_{1}, X_{2} ; W_{1}, W_{2}\right),
$$

where the poles of the coefficients were computed in section 4.2,

$$
\begin{aligned}
& a_{2}(\nu)=\frac{1}{\nu^{2}+(\Delta-h)^{2}}, \quad a_{1}(\nu)=-\frac{2}{\Delta(2 h-\Delta)\left(\nu^{2}+(h+1)^{2}\right)}, \\
& a_{0}(\nu)=\frac{1}{2 h(\Delta-1)(2 h-\Delta-1)\left(\nu^{2}+(h+1)^{2}\right)}-\frac{1}{2 h \Delta(2 h-\Delta)\left(\nu^{2}+h^{2}\right)} .
\end{aligned}
$$

We will show that this is the complete expression for these coefficients. In addition to the traceless part of the propagator, we add a second term which is a pure trace,

$$
\left(\eta^{A B}+X_{1}^{A} X_{1}^{B}\right)\left(\eta^{C D}+X_{2}^{C} X_{2}^{D}\right) \int d \nu t(\nu) \Omega_{\nu, 0}\left(X_{1}, X_{2}\right)
$$


and a third term given by

$$
\left[\left(\eta^{A B}+X_{1}^{A} X_{1}^{B}\right) \nabla_{2}^{C} \nabla_{2}^{D}+\nabla_{1}^{A} \nabla_{1}^{B}\left(\eta^{C D}+X_{2}^{C} X_{2}^{D}\right)\right] \int d \nu q(\nu) \Omega_{\nu, 0}\left(X_{1}, X_{2}\right) .
$$

We now use the full equation of motion (3.4) to write the complete equation for the propagator

$$
\begin{aligned}
& \left(D_{1}^{2}+2-\Delta(\Delta-d)\right) G_{\mu_{1} \mu_{2} ; \nu_{1} \nu_{2}}-D_{\mu_{1}} D^{\sigma} G_{\sigma \mu_{2} ; \nu_{1} \nu_{2}}-D_{\mu_{2}} D^{\sigma} G_{\mu_{1} \sigma ; \nu_{1} \nu_{2}}-2 g_{\mu_{1} \mu_{2}} G_{\sigma}^{\sigma} ; \nu_{1} \nu_{2} \\
+ & \left(D_{\mu_{1}} D_{\mu_{2}}-\frac{\Delta(\Delta-d)}{d-1} g_{\mu_{1} \mu_{2}}\right) G_{\sigma}^{\sigma}{ }_{; \nu_{1} \nu_{2}}=-\frac{1}{2}\left(g_{\mu_{1} \nu_{1}} g_{\mu_{2} \nu_{2}}+g_{\mu_{1} \nu_{2}} g_{\mu_{2} \nu_{1}}-\frac{2 g_{\mu_{1} \mu_{2}} g_{\nu_{1} \nu_{2}}}{d-1}\right) \delta\left(x_{1}, x_{2}\right) .
\end{aligned}
$$

We can determine the coefficients $t(\nu)$ and $q(\nu)$ by imposing this equation, including contact terms. To see this, let us apply the left hand side to each one of the terms (4.27), (4.30) and (4.31), that make up the full propagator. Acting on the traceless part of the propagator, the left hand side of (4.32) gives a traceless contribution

$$
-\left(W_{12}\right)^{2} \delta\left(X_{1}, X_{2}\right)+\frac{(2 h-1)^{2}\left(\left(W_{1} \cdot \nabla_{1}\right)\left(W_{2} \cdot \nabla_{2}\right)\right)^{2}}{2 h(2 h+1) \Delta(\Delta-1)(2 h-\Delta)(2 h-\Delta-1)} \delta\left(X_{1}, X_{2}\right),
$$

plus a contribution with non zero trace,

$$
\int d \nu \rho(\nu)\left(\eta^{A B}+X_{1}^{A} X_{1}^{B}\right)\left[\nabla_{2}^{C} \nabla_{2}^{D}+\frac{\nu^{2}+h^{2}}{2 h+1}\left(\eta^{C D}+X_{2}^{C} X_{2}^{D}\right)\right] \Omega_{\nu, 0}\left(X_{1}, X_{2}\right),
$$

where

$$
\rho(\nu)=\frac{2}{(2 h+1) \Delta(2 h-\Delta)}-\frac{2(2 h-1)\left(\nu^{2}+h^{2}\right)}{(2 h+1)^{2} \Delta(\Delta-1)(2 h-\Delta)(2 h-\Delta-1)} .
$$

Applying the left hand side of (4.32) to (4.30), we obtain

$$
\int d \nu t(\nu)\left[\sigma(\nu)\left(\eta^{A B}+X_{1}^{A} X_{1}^{B}\right)+(2 h-1) \nabla_{1}^{A} \nabla_{1}^{B}\right]\left(\eta^{C D}+X_{2}^{C} X_{2}^{D}\right) \Omega_{\nu, 0}\left(X_{1}, X_{2}\right),
$$

where

$$
\sigma(\nu)=\frac{4 h(\Delta-1)(2 h-\Delta-1)}{2 h-1}-\nu^{2}-h^{2} .
$$

Finally, the left hand side of (4.32) applied to (4.31) gives

$$
\begin{aligned}
\int d \nu q(\nu) & \left(\left[\sigma(\nu)\left(\eta^{A B}+X_{1}^{A} X_{1}^{B}\right)+(2 h-1) \nabla_{1}^{A} \nabla_{1}^{B}\right] \nabla_{2}^{C} \nabla_{2}^{D}\right. \\
& \left.+\Delta(2 h-\Delta)\left[\nabla_{1}^{A} \nabla_{1}^{B}-\frac{\nu^{2}+h^{2}}{2 h-1}\left(\eta^{A B}+X_{1}^{A} X_{1}^{B}\right)\right]\left(\eta^{C D}+X_{2}^{C} X_{2}^{D}\right)\right) \Omega_{\nu, 0}\left(X_{1}, X_{2}\right) .
\end{aligned}
$$

To perform these calculations the following identities were useful

$$
\begin{aligned}
& \nabla^{2} \nabla^{A} \nabla^{B} F(X)=\nabla^{A} \nabla^{B}\left[\nabla^{2}-2(2 h+1)\right] F(X)+2\left(\eta^{A B}+X^{A} X^{B}\right) \nabla^{2} F(X), \\
& \nabla^{A} \nabla_{C} \nabla^{C} \nabla^{B} F(X)+\nabla^{B} \nabla_{C} \nabla^{A} \nabla^{C} F(X)=\nabla^{A} \nabla^{B}\left(2 \nabla^{2}-4 h\right) F(X),
\end{aligned}
$$

where $F(X)$ is a scalar function in AdS and the covariant derivative was defined in (2.21). 
Putting together the contributions from the three terms and requiring that they sum up to the right hand side of (4.32), determines

$$
\begin{aligned}
q(\nu) & =-\frac{2 h-1}{2 h(2 h+1) \Delta(\Delta-1)(2 h-\Delta)(2 h-\Delta-1)}, \\
t(\nu) & =q(\nu)\left[\frac{\nu^{2}+h^{2}}{2 h+1}-\frac{\Delta(2 h-\Delta)}{2 h-1}\right] .
\end{aligned}
$$

Since both $t(\nu)$ and $q(\nu)$ are analytic in $\nu$, we conclude that the terms (4.30) and (4.31) are pure contact terms, as expected.

It should be possible to generalize this analysis to propagators with higher spin, using the appropriate equations of motion [33, 34, 39-41]. However, we will not attempt here to find a closed formula for the contact terms of a propagator of arbitrary spin.

\subsubsection{Graviton propagator}

In the massless limit, the bulk propagator couples to a conserved current. This means one can drop total derivatives of the propagator. The full propagator is then given by

$$
\begin{aligned}
& \int d \nu a_{2}(\nu) \Omega_{\nu, 2}^{A B, C B}\left(X_{1}, X_{2}\right)+(\text { total derivative }) \\
& +\left(\eta^{A B}+X_{1}^{A} X_{1}^{B}\right)\left(\eta^{C D}+X_{2}^{C} X_{2}^{D}\right) \int d \nu\left[t(\nu)+a_{0}(\nu) \frac{\left(h^{2}+\nu^{2}\right)^{2}}{(2 h+1)^{2}}\right] \Omega_{\nu, 0}\left(X_{1}, X_{2}\right) .
\end{aligned}
$$

The graviton propagator is obtained in the massless limit $\Delta \rightarrow d=2 h$. Recall that there is no van Dam-Veltman-Zhakarov discontinuity in $\operatorname{AdS}[42,43]$. In this limit, both $t(\nu)$ and $a_{0}(\nu)$ diverge but the combination that appears in (4.41) remains finite. This gives the split representation of the graviton propagator

$$
\begin{aligned}
\Pi_{d, 2}^{A B, C D}\left(X_{1}, X_{2}\right)= & \int \frac{d \nu}{\nu^{2}+h^{2}} \Omega_{\nu, 2}^{A B, C D}\left(X_{1}, X_{2}\right)+(\text { total derivative }) \\
& -\frac{\left(\eta^{A B}+X_{1}^{A} X_{1}^{B}\right)\left(\eta^{C D}+X_{2}^{C} X_{2}^{D}\right)}{2 h(2 h-1)} \int \frac{d \nu}{(h+1)^{2}+\nu^{2}} \Omega_{\nu, 0}\left(X_{1}, X_{2}\right) .
\end{aligned}
$$

Notice that the total derivative (or pure gauge term) diverges in the massless limit.

\section{Three-point function}

A simple application of the above formalism is to consider a three-point Witten diagram with two insertions of scalar fields $\phi_{1}, \phi_{2}$ and one of a spin $J$ field at the boundary, as represented in figure 4. This diagram, computed at tree level, will allow us to related the OPE coefficient of the dual operators in the field theory to the local coupling of AdS fields. The simplest AdS local cubic vertex of a spin $J$ field to two scalars $\phi_{1}$ and $\phi_{2}$ is of the form

$$
g_{\phi_{1} \phi_{2} h} \int_{\mathrm{AdS}} d x \sqrt{g}\left(\phi_{2} \nabla_{\mu_{1}} \cdots \nabla_{\mu_{J}} \phi_{1}\right) h^{\mu_{1} \cdots \mu_{J}},
$$

where $g_{\phi_{1} \phi_{2} h}$ is a bulk coupling constant. Notice that the derivatives can act on either of the scalar fields because we consider a spin $J$ field of vanishing divergence. Moreover, a 


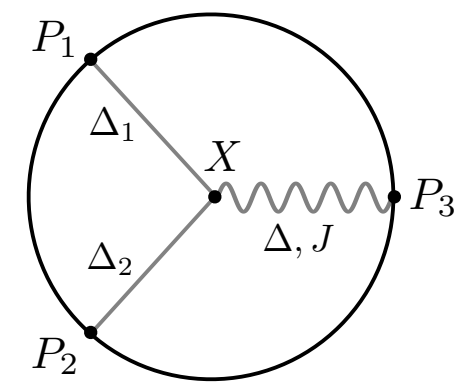

Figure 4. Witten diagram that computes a CFT three-point function of a spin $J$ primary operator of dimension $\Delta$ and two scalar primary operators of dimension $\Delta_{1}$ and $\Delta_{2}$.

vertex with more derivatives can be reduced to this form by integrating by parts and using the (linear) equations of motion of the fields.

To compute the cubic Witten diagram in figure 4 from the above vertex, we consider insertions of the scalar field $\phi_{1}$ at the boundary point $P_{1}$, of $\phi_{2}$ at $P_{2}$ and of the spin $J$ field at $P_{3}$. The corresponding bulk-to-boudary propagator for a field of dimension $\Delta$ and spin $J$ is given in (3.39). Thus, this Witten diagram is given by the integral over the AdS interaction point of the bulk-to-boudary propagators, and generates the CFT three-point function $^{3}$

$$
\begin{aligned}
& \left\langle\mathcal{O}_{\phi_{1}}\left(P_{1}\right) \mathcal{O}_{\phi_{2}}\left(P_{2}\right) \mathcal{O}_{h}\left(P_{3}, Z\right)\right\rangle \\
& =\frac{g_{\phi_{1} \phi_{2} h}}{\sqrt{\mathcal{C}_{\Delta_{1}} \mathcal{C}_{\Delta_{2}} \mathcal{C}_{\Delta, J}}} \int_{\mathrm{AdS}} d X \Pi_{\Delta_{2}, 0}\left(X, P_{2}\right) \frac{\Pi_{\Delta, J}\left(X, P_{3} ; K, Z\right)(W \cdot \nabla)^{J} \Pi_{\Delta_{1}, 0}\left(X, P_{1}\right)}{J !\left(\frac{d-1}{2}\right)_{J}}
\end{aligned}
$$

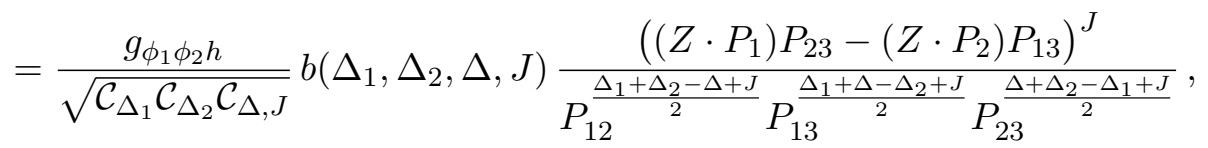

where $K$ is the projector defined in (2.12) and we used the notation $P_{i j}=-2 P_{i} \cdot P_{j}$ and $\mathcal{C}_{\Delta}=\mathcal{C}_{\Delta, 0}$ for short. In the last equality, we used the fact that this three-point function is determined by conformal symmetry up to an overall constant. To determine the constant $b\left(\Delta_{1}, \Delta_{2}, \Delta, J\right)$ we have to perform the integral over AdS. In the case $J=0$, the AdS integral in (5.2) is well known $[44,45]$ and gives

$$
b\left(\Delta_{1}, \Delta_{2}, \Delta, 0\right)=\mathcal{C}_{\Delta_{1}} \mathcal{C}_{\Delta_{2}} \mathcal{C}_{\Delta} \frac{\pi^{\frac{d}{2}} \Gamma\left(\frac{\Delta_{1}+\Delta_{2}+\Delta-d}{2}\right) \Gamma\left(\frac{\Delta_{1}+\Delta_{2}-\Delta}{2}\right) \Gamma\left(\frac{\Delta_{1}+\Delta-\Delta_{2}}{2}\right) \Gamma\left(\frac{\Delta+\Delta_{2}-\Delta_{1}}{2}\right)}{2 \Gamma\left(\Delta_{1}\right) \Gamma\left(\Delta_{2}\right) \Gamma(\Delta)} .
$$

To compute the integral for general spin $J$, we use the differential operator

$$
D_{31}=Z \cdot P_{1}\left(Z \cdot \frac{\partial}{\partial Z}-P_{3} \cdot \frac{\partial}{\partial P_{3}}\right)+P_{3} \cdot P_{1}\left(Z \cdot \frac{\partial}{\partial P_{3}}\right)
$$

\footnotetext{
${ }^{3}$ The factor $1 / \sqrt{\mathcal{C}_{\Delta_{1}} \mathcal{C}_{\Delta_{2}} \mathcal{C}_{\Delta, J}}$ corresponds to the normalization choice of CFT operators that have unit two-point function,

$$
\left\langle\mathcal{O}\left(P_{1}, Z_{1}\right) \mathcal{O}\left(P_{2}, Z_{2}\right)\right\rangle=\frac{\left(\left(-2 P_{1} \cdot P_{2}\right)\left(Z_{2} \cdot Z_{1}\right)+2\left(Z_{2} \cdot P_{1}\right)\left(Z_{1} \cdot P_{2}\right)\right)^{J}}{\left(-2 P_{1} \cdot P_{2}\right)^{\Delta+J}} .
$$
}


introduced in [13]. Noting that

$$
\begin{aligned}
& \frac{1}{J !\left(\frac{d-1}{2}\right)_{J}} \Pi_{\Delta, J}\left(X, P_{3} ; K, Z\right)(W \cdot \nabla)^{J} \Pi_{\Delta_{1}, 0}\left(X, P_{1}\right) \\
= & 2^{J} \mathcal{C}_{\Delta_{1}} \mathcal{C}_{\Delta, J} \frac{\left(\Delta_{1}\right)_{J}}{(\Delta)_{J}}\left(D_{31}\right)^{J} \frac{1}{\left(-2 P_{3} \cdot X\right)^{\Delta}\left(-2 P_{1} \cdot X\right)^{\Delta_{1}+J}},
\end{aligned}
$$

the computation of the above AdS integral reduces to that of the scalar case by commuting the differential operator $\left(D_{31}\right)^{J}$ with the integral symbol. Finally, using

$$
\begin{aligned}
& \frac{\left(\left(Z \cdot P_{1}\right) P_{23}-\left(Z \cdot P_{2}\right) P_{13}\right)^{J}}{P_{12}^{\frac{\Delta_{1}+\Delta_{2}-\Delta+J}{2}} P_{13}^{\frac{\Delta_{1}+\Delta-\Delta_{2}+J}{2}}} P_{23}^{\frac{\Delta+\Delta_{2}-\Delta_{1}+J}{2}} \\
= & \frac{1}{\left(\frac{\Delta+\Delta_{2}-\Delta_{1}-J}{2}\right)_{J}}\left(D_{31}\right)^{J} \frac{1}{P_{12}^{\frac{\Delta_{1}+\Delta_{2}-\Delta+J}{2}}} P_{13}^{\frac{\Delta_{1}+\Delta-\Delta_{2}-J}{2}} P_{23}^{\frac{\Delta+\Delta_{2}-\Delta_{1}+J}{2}},
\end{aligned}
$$

we arrive at the result

$$
\begin{aligned}
& b\left(\Delta_{1}, \Delta_{2}, \Delta, J\right)=2^{J}\left(\frac{\Delta+\Delta_{2}-\Delta_{1}-J}{2}\right)_{J} \frac{\mathcal{C}_{\Delta, J}}{\mathcal{C}_{\Delta, 0}} \frac{\mathcal{C}_{\Delta_{1}}}{\mathcal{C}_{\Delta_{1}+J}} \frac{\left(\Delta_{1}\right)_{J}}{(\Delta)_{J}} b\left(\Delta_{1}+J, \Delta_{2}, \Delta, 0\right) \\
= & \mathcal{C}_{\Delta_{1}} \mathcal{C}_{\Delta_{2}} \mathcal{C}_{\Delta, J} \frac{\pi^{\frac{d}{2}} \Gamma\left(\frac{\Delta_{1}+\Delta_{2}+\Delta-d+J}{2}\right) \Gamma\left(\frac{\Delta_{1}+\Delta_{2}-\Delta+J}{2}\right) \Gamma\left(\frac{\Delta+\Delta_{1}-\Delta_{2}+J}{2}\right) \Gamma\left(\frac{\Delta+\Delta_{2}-\Delta_{1}+J}{2}\right)}{2^{1-J} \Gamma\left(\Delta_{1}\right) \Gamma\left(\Delta_{2}\right) \Gamma(\Delta+J)} .
\end{aligned}
$$

The result (5.2) establishes the relation between the local AdS coupling $g_{\phi_{1} \phi_{2} h}$ and the CFT OPE coefficient $C_{\phi_{1} \phi_{2} h}$,

$$
C_{\phi_{1} \phi_{2} h}=\frac{b\left(\Delta_{1}, \Delta_{2}, \Delta, J\right)}{\sqrt{\mathcal{C}_{\Delta_{1}} \mathcal{C}_{\Delta_{2}} \mathcal{C}_{\Delta, J}}} g_{\phi_{1} \phi_{2} h} .
$$

As a check of this result, let us consider the case of the stress-energy tensor with $\Delta=d$ and $J=2$. In this case, the OPE coefficient is determined by a Ward identity $[46,47]$

$$
C_{\phi \phi T_{\mu \nu}}=\frac{d \Delta_{\phi}}{(d-1) \sqrt{C_{T}}}
$$

where $C_{T}$ is the coefficient of the two point function of the (standard) stress tensor (notice that here we are redefining the stress tensor such that it has unit two point function). This is given by $[48,49]$

$$
C_{T}=\frac{1}{2 \pi G_{N}} \frac{d+1}{d-1} \frac{\pi^{\frac{d}{2}} \Gamma(d+1)}{\Gamma^{3}\left(\frac{d}{2}\right)},
$$

where $G_{N}$ is the gravitational coupling of the $(d+1)$-dimensional (Euclidean) dual theory

$$
S_{E}=\frac{1}{16 \pi G_{N}} \int d^{d+1} x \sqrt{g}\left[-d(d-1)-\mathcal{R}+\frac{1}{2}(\nabla \phi)^{2}+\frac{1}{2} M_{\phi}^{2} \phi^{2}\right],
$$

and we are setting the AdS radius to one. Expanding this action around the AdS background, $g_{\mu \nu}=g_{\mu \nu}^{\mathrm{AdS}}+\sqrt{32 \pi G_{N}} h_{\mu \nu}$, and rescaling the scalar field $\phi \rightarrow \sqrt{16 \pi G_{N}} \phi$, we obtain canonically normalized kinetic terms $\frac{1}{2}(\nabla \phi)^{2}$ for the scalar and (3.3) with $M^{2}=-2$ for 
$h_{\mu \nu}$. This means that the cubic coupling is given by $g_{\phi \phi h_{\mu \nu}}=\sqrt{8 \pi G_{N}}$. Substituting this value in equation (5.8) and multiplying by 2 because $\phi_{1}=\phi_{2},{ }^{4}$ we indeed confirm the Ward identity (5.9) for generic dimension $d$.

Let us now specify to the case of planar $\mathcal{N}=4$ SYM at large 't Hooft coupling $\lambda$ and use its dual description as type IIB superstring theory on $\mathrm{AdS}_{5} \times S^{5}$. The string theory action takes the schematic form

$$
S=\frac{1}{2 \kappa_{10}^{2}} \int d^{10} x \sqrt{g}\left[\mathcal{L}_{2}+\ell_{s}^{2} \mathcal{L}_{4}+\ldots\right]
$$

where $\mathcal{L}_{k}$ is the part of the Lagrangian density with $k$ spacetime derivatives, $\kappa_{10}$ is the gravitational coupling and $\ell_{s}$ is the string length. Expanding around the $\mathrm{AdS}_{5} \times S^{5}$ background and reducing to $\mathrm{AdS}_{5}$, the effective action becomes

$$
S=\frac{1}{2 \kappa^{2}} \int d^{5} x \sqrt{g}\left[\ell_{s}^{-2} \widetilde{\mathcal{L}}_{0}+\widetilde{\mathcal{L}}_{2}+\ell_{s}^{2} \widetilde{\mathcal{L}}_{4}+\ldots\right]
$$

where $\widetilde{\mathcal{L}}_{k}$ contains $k$ or less spacetime derivatives (notice that some derivatives in the tendimensional action can act on background fields and produce factors of $1 / R$ which is 1 in our units). The gravitational coupling $\kappa$ satisfies $\kappa^{2}=8 \pi G_{N}^{(5)}$ and can be removed by rescaling the fields so that they have canonically normalized kinetic terms in the action. This gives the following scaling for the cubic coupling of the type (5.1),

$$
g_{\phi_{1} \phi_{2} h} \sim \kappa\left[\ell_{s}^{J-2}+O\left(\ell_{s}^{J}\right)\right] .
$$

Converting to gauge theory parameters, the planar OPE coefficient for operators with unit two point function will then be given by

$$
C_{\phi_{1} \phi_{2} h} \sim \frac{1}{N}\left(\frac{1}{\lambda}\right)^{\frac{J-2}{4}} \frac{b\left(\Delta_{1}, \Delta_{2}, \Delta, J\right)}{\sqrt{\mathcal{C}_{\Delta_{1}} \mathcal{C}_{\Delta_{2}} \mathcal{C}_{\Delta, J}}}\left[1+O\left(\frac{1}{\sqrt{\lambda}}\right)\right] .
$$

Note that the explicit dependence on the 't Hooft coupling comes from the tree level string theory coupling of the dual fields. There is additionally an implicit dependence on the 't Hooft coupling through the dimension of the operators that are not protected, in general. For example, in the case of two protected operators (fixed $\Delta_{1}$ and $\Delta_{2}$ ) and a non-protected operator with $\Delta(\lambda) \sim \lambda^{1 / 4}$ and fixed $J$ we find

$$
C_{\phi_{1} \phi_{2} h} \sim \frac{1}{N} \frac{\lambda^{\frac{\Delta_{1}+\Delta_{2}-1}{4}} 2^{-\Delta(\lambda)}}{\sin \left(\frac{\pi}{2}\left(\Delta_{1}+\Delta_{2}+J-\Delta(\lambda)\right)\right)} .
$$

The presence of poles (as a function of $\lambda$ ) in the 3pt-functions as been previously observed in [50] using Witten diagrams and in [51] using a large spin expansion that is valid to all orders in planar perturbation theory.

\footnotetext{
${ }^{4}$ Notice that there is an extra Wick contraction in this case.
} 


\section{Four-point function}

As a further application if the embedding formalism we consider four-point functions of scalar primary operators computed from Witten diagrams with a spin $J$ field exchange. In general, a four-point function of scalar primary operators in a conformal field theory can be decomposed in partial waves as follows [52]

$$
\left\langle\mathcal{O}_{\phi_{1}} \ldots \mathcal{O}_{\phi_{4}}\right\rangle=\frac{1}{\left(P_{12}\right)^{\frac{\Delta_{1}+\Delta_{2}}{2}}\left(P_{34}\right)^{\frac{\Delta_{3}+\Delta_{4}}{2}}}\left(\frac{P_{24}}{P_{14}}\right)^{\frac{\Delta_{12}}{2}}\left(\frac{P_{14}}{P_{13}}\right)^{\frac{\Delta_{34}}{2}} \sum_{l=0}^{\infty} \int_{-\infty}^{\infty} d \nu b_{l}(\nu) F_{\nu, l}(u, v),
$$

where the conformal partial wave $F_{\nu, l}(u, v)$ is a function of the cross ratios

$$
u=\frac{P_{12} P_{34}}{P_{13} P_{24}}, \quad v=\frac{P_{14} P_{23}}{P_{13} P_{24}} .
$$

This function can be defined as the integral of the product of two three-point functions

$$
\begin{aligned}
& \left(P_{12}\right)^{-\frac{\Delta_{1}+\Delta_{2}}{2}}\left(P_{34}\right)^{-\frac{\Delta_{3}+\Delta_{4}}{2}}\left(\frac{P_{24}}{P_{14}}\right)^{\frac{\Delta_{12}}{2}}\left(\frac{P_{14}}{P_{13}}\right)^{\frac{\Delta_{34}}{2}} F_{\nu, l}(u, v) \\
= & \frac{1}{\beta} \int d P_{5}\left\langle\mathcal{O}_{\phi_{1}}\left(P_{1}\right) \mathcal{O}_{\phi_{2}}\left(P_{2}\right) \mathcal{O}_{h+i \nu, l}\left(P_{5}, D_{Z}\right)\right\rangle\left\langle\mathcal{O}_{h-i \nu, l}\left(P_{5}, Z\right) \mathcal{O}_{\phi_{3}}\left(P_{3}\right) \mathcal{O}_{\phi_{4}}\left(P_{4}\right)\right\rangle,
\end{aligned}
$$

where $\beta$ is a normalization constant given in equation (E.7) of appendix $\mathrm{E}$ and $D_{Z}$ is the differential operator that implements index contraction defined in (4.2). In this expression, the three-point functions are given by

$$
\left\langle\mathcal{O}_{\phi_{1}}\left(P_{1}\right) \mathcal{O}_{\phi_{2}}\left(P_{2}\right) \mathcal{O}_{h+i \nu, l}\left(P_{5}, Z\right)\right\rangle=\frac{\left(\left(Z \cdot P_{1}\right) P_{25}-\left(Z \cdot P_{2}\right) P_{15}\right)^{l}}{P_{15}^{\frac{\Delta_{1}+h+i \nu-\Delta_{2}+l}{2}} P_{25}^{\frac{\Delta_{2}+h+i \nu-\Delta_{1}+l}{2}} P_{12}^{\frac{\Delta_{1}+\Delta_{2}+h-i \nu+l}{2}}} .
$$

We remark that the conformal partial wave $F_{\nu, l}(u, v)$ can also be written as a conformal block of dimension $h+i \nu$ and spin $l$ plus the conformal block of its shadow operator, which has dimension $h-i \nu$ and the same spin. ${ }^{5}$

As an application of the technology developed in the previous sections, we will compute the conformal partial wave decomposition of the four-point function associated to the Witten diagram of figure 5. In this diagram, the external operators are scalar fields $\phi_{i}$, with dimension $\Delta_{i}$, which exchange a field of dimension $\Delta$ and spin $J$. The spin $J$ field couples to the external scalars through the cubic coupling (5.1). Next we use the split representation of the spin $J$ bulk-to-bulk propagator given by (4.1) and (4.13),

$$
\begin{aligned}
& \frac{g_{\phi_{1} \phi_{2} h} g_{\phi_{3} \phi_{4} h}}{\left[J !\left(\frac{d-1}{2}\right)_{J}\right]^{2}} \int d X_{1} d X_{2} \Pi_{\Delta_{1}}\left(P_{1}, X_{1}\right)\left[\left(K_{1} \cdot \nabla_{1}\right)^{J} \Pi_{\Delta_{2}}\left(P_{2}, X_{1}\right)\right] \Pi_{\Delta_{3}}\left(P_{3}, X_{2}\right) \\
& {\left[\left(K_{2} \cdot \nabla_{2}\right)^{J} \Pi_{\Delta_{4}}\left(P_{4}, X_{2}\right)\right] \sum_{l=0}^{J} \int d \nu a_{l}(\nu)\left(W_{1} \cdot \nabla_{1}\right)^{J-l}\left(W_{2} \cdot \nabla_{2}\right)^{J-l} \Omega_{\nu, l}\left(X_{1}, X_{2} ; W_{1}, W_{2}\right),}
\end{aligned}
$$

\footnotetext{
${ }^{5}$ The conformal partial wave $F_{\nu, J}(u, v)$ can be expressed in terms of conformal blocks $G_{\Delta, J}(u, v)$ as

$$
F_{\nu, J}(u, v)=\kappa_{\nu, J} G_{h+i \nu, J}(u, v)+\kappa_{-\nu, J} G_{h-i \nu, J}(u, v),
$$

where $\kappa_{\nu, J}$ is a normalization constant defined in [53].
} 

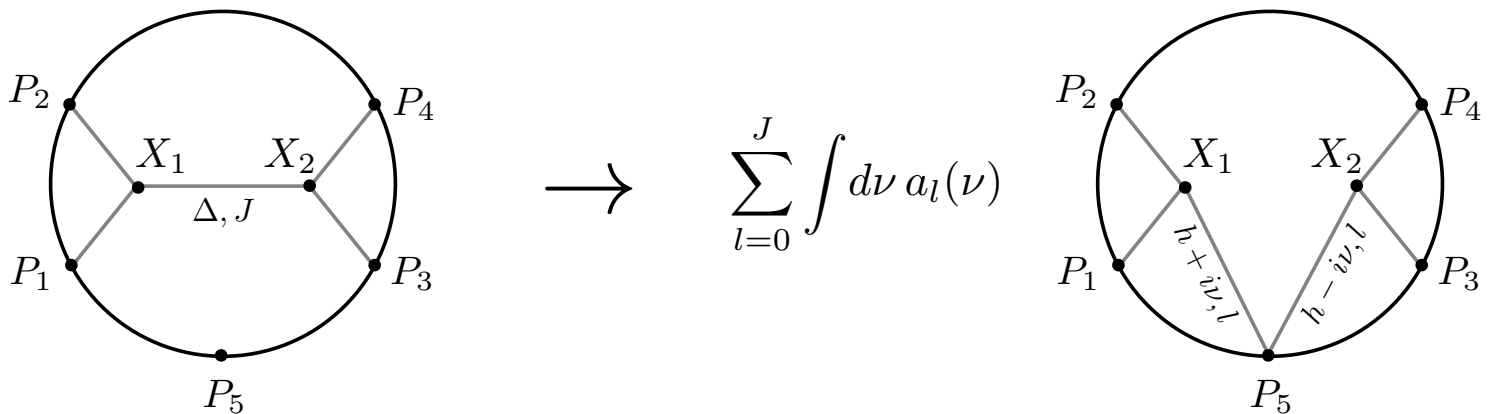

Figure 5. Witten diagram describing a spin $J$ exchange between scalar primaries of arbitrary dimension. Using the split representation of the bulk-to-bulk propagator this diagram can be converted into the product of two three-point functions integrated over the common boundary point $P_{5}$.

where $g_{\phi_{1} \phi_{2} h}$ and $g_{\phi_{3} \phi_{4} h}$ are the cubic couplings between the external scalars and the spin $J$ field. The corresponding diagram is also represented in figure 5. The integration over the bulk points $X_{1}$ and $X_{2}$ produces a product of two three-point functions integrated over the point $P_{5}$ exactly as in the definition of the conformal partial wave $F_{\nu, J}$. Therefore, we conclude that the coefficients $b_{l}(\nu)$ of the partial wave expansion (6.1) are proportional to the functions $a_{l}(\nu)$ of the split representation (4.27) of the propagator. More precisely, we can write

$$
b_{l}(\nu)=g_{\phi_{1} \phi_{2} h} g_{\phi_{1} \phi_{2} h} \alpha_{l}(\nu) a_{l}(\nu)
$$

where $\alpha_{l}(\nu)$ is independent of $\Delta$ and it is given in equation (E.8) of appendix E.

The structure of the coefficients $b_{l}(\nu)$ was studied in detail in appendix A.5 of [53]. In particular, the residues of the spurious poles of $b_{l}(\nu)$ are related through

$$
b_{l}(\nu) \approx-\frac{\mathcal{Z}_{l+q, q} b_{l+q}(i(h-1+l))}{\nu^{2}+(h+l+q-1)^{2}}, \quad q=1,2, \ldots,
$$

where

$$
\mathcal{Z}_{J, q}=\frac{J !}{(J-q) ! q !} \frac{2(-2)^{q}\left(\frac{\Delta_{1}+\Delta_{2}+1-2 h-q}{2}\right)_{q}\left(\frac{\Delta_{3}+\Delta_{4}+1-2 h-q}{2}\right)_{q}\left(\frac{\Delta_{12}+1-q}{2}\right)_{q}\left(\frac{\Delta_{34}+1-q}{2}\right)_{q}}{\Gamma(q)(h+J-q)_{q-1}} .
$$

In equation $(6.8)$ we used the symbol $\approx$ to mean that the two sides of the expression have the same residue at the pole in $\nu^{2}$ shown explicitly. Given (6.7) and (6.8) it is possible to derive a similar constraint on the coefficients $a_{l}(\nu)$,

$$
\begin{aligned}
a_{l}(\nu) & \approx-\frac{\mathcal{Z}_{l+q, q} \alpha_{l+q}(i(h+l-1))}{\alpha_{l}(i(h+q+l-1))} \frac{a_{l+q}(i(h-1+l))}{\nu^{2}+(h+l+q-1)^{2}} \\
& =\frac{(l+q) !}{l ! q !} \frac{(-1)^{q+1}}{2^{q-1}(q-1) !(h+l)_{q-1}} \frac{a_{l+q}(i(h-1+l))}{\nu^{2}+(h+l+q-1)^{2}},
\end{aligned}
$$


where the last expression was guessed by generalizing the results of explicit calculations for $l=0, \ldots, 20$ and $q=0, \ldots, 20$. It is remarkable that all the dependence of the functions $\mathcal{Z}_{J, q}, \alpha_{l}$ and $\alpha_{l+q}$ on the external dimensions $\Delta_{i}$ cancelled. This had to happen because $a_{l}(\nu)$ are the expansion coefficients of the bulk propagator in the split representation. This is a very non-trivial consistency check of our results.

In fact, using (6.10) we can obtain the full split representation of a dimension $\Delta$ and spin $J$ bulk-to-bulk propagator. The starting point is $a_{l}(\nu)=0$ for $l>J$ and

$$
a_{J}(\nu)=\frac{1}{\nu^{2}+(\Delta-h)^{2}},
$$

as derived in section 4 . Then, for $l<J$ we take the minimal choice that is compatible with (6.10),

$$
a_{l}(\nu)=\sum_{q=1}^{J-l} \frac{(l+q) !}{l ! q !} \frac{(-1)^{q+1}}{2^{q-1}(q-1) !(h+l)_{q-1}} \frac{a_{l+q}(i(h-1+l))}{\nu^{2}+(h+l+q-1)^{2}} .
$$

One can easily check that this reproduces the results (4.23) and (4.24) for $l=J-1$ and $l=J-2$. Moreover, one can check that this is consistent with the explicit expression (4.19) for the coefficients $a_{l}(\nu)$ found in section 4.2.

\subsection{Example: AdS graviton exchange}

One application of the split representation derived above is the computation of the AdS graviton exchange diagram. More precisely, we compute the contribution to the four-point function of scalar primary operators, from the diagram in figure 5(a), where the exchanged bulk field is the graviton. This reads ${ }^{6}$

$$
\begin{aligned}
& \left\langle\mathcal{O}_{\phi_{1}}\left(P_{1}\right) \mathcal{O}_{\phi_{1}}\left(P_{2}\right) \mathcal{O}_{\phi_{3}}\left(P_{3}\right) \phi_{3}\left(P_{4}\right)\right\rangle \\
= & \frac{8 \pi G_{N}}{\mathcal{C}_{\Delta_{1}} \mathcal{C}_{\Delta_{3}}} \int_{\text {AdS }} d X_{1} d X_{2} T_{A B}^{(12)}\left(X_{1}\right) \Pi_{d, 2}^{A B, C D}\left(X_{1}, X_{2}\right) T_{C D}^{(34)}\left(X_{2}\right),
\end{aligned}
$$

where

$$
\begin{aligned}
& T_{A B}^{(12)}(X)=\nabla_{A} \Pi_{\Delta_{1}}\left(X, P_{1}\right) \nabla_{B} \Pi_{\Delta_{1}}\left(X, P_{2}\right)+\nabla_{B} \Pi_{\Delta_{1}}\left(X, P_{1}\right) \nabla_{A} \Pi_{\Delta_{1}}\left(X, P_{2}\right) \\
& -\left(\eta_{A B}+X_{A} X_{B}\right)\left[\nabla^{C} \Pi_{\Delta_{1}}\left(X, P_{1}\right) \nabla_{C} \Pi_{\Delta_{1}}\left(X, P_{2}\right)+\Delta_{1}\left(\Delta_{1}-d\right) \Pi_{\Delta_{1}}\left(X, P_{1}\right) \Pi_{\Delta_{1}}\left(X, P_{2}\right)\right],
\end{aligned}
$$

and similarly for $T_{C D}^{(34)}(X)$. This Witten diagram was first computed in [54] for some specific values of $\Delta_{1}, \Delta_{3}$ and $d$. Here, we will use the split representation (4.42) of the graviton propagator to obtain directly the conformal partial wave expansion of the Witten diagram for all values of $\Delta_{1}, \Delta_{3}$ and spacetime dimension $d=2 h$. This calculation is very similar to the one discussed above for (6.6). Since the the sources $T_{A B}^{(12)}\left(X_{1}\right)$ and $T_{C D}^{(34)}\left(X_{2}\right)$ are conserved we can drop the total derivative terms in (4.42), as expected. To determine the contribution from the remaining two terms, we use the representation (4.1) of the harmonic

\footnotetext{
${ }^{6}$ We include the denominator $\mathcal{C}_{\Delta_{1}} \mathcal{C}_{\Delta_{3}}$ in order to obtain the four-point function of operators normalized to have unit two-point function (see footnote 3 ).
} 
functions $\Omega_{\nu, 2}$ and $\Omega_{\nu, 0}$. After integrating over the bulk points $X_{1}$ and $X_{2}$, we are left with the integral over the boundary point $P$ in (4.1), of the product of two three-point functions like in the definition (6.3) of the conformal partial waves. Then the partial amplitudes, as defined in (6.1), are given by

$$
\begin{aligned}
& b_{2}(\nu)=\frac{8 \pi G_{N}}{2 \pi^{h} \Gamma\left(\Delta_{1}\right) \Gamma\left(\Delta_{3}\right) \Gamma\left(\Delta_{1}+1-h\right) \Gamma\left(\Delta_{3}+1-h\right)} \frac{1}{\nu^{2}+h^{2}}, \\
& b_{0}(\nu)=-8 \pi G_{N} \frac{\left[4 \Delta_{1}\left(2 h-\Delta_{1}\right)+(2 h-1)\left(h^{2}+\nu^{2}\right)\right]\left[4 \Delta_{3}\left(2 h-\Delta_{3}\right)+(2 h-1)\left(h^{2}+\nu^{2}\right)\right]}{64 \pi^{h} h(2 h-1) \Gamma\left(\Delta_{1}+1-h\right) \Gamma\left(\Delta_{3}+1-h\right) \Gamma\left(\Delta_{1}\right) \Gamma\left(\Delta_{3}\right)\left[\nu^{2}+(h+1)^{2}\right]}
\end{aligned}
$$

which determine the conformal partial wave expansion of the graviton exchange diagram. Notice that this result is consistent with the relation (6.8) for the spurious poles of the partial amplitudes. Moreover, the pole of $b_{2}(\nu)$ is fixed by the conformal Ward identity. To see that, we first use the relation between the residues of the conformal partial amplitudes $b_{l}(\nu)$ and OPE coefficients $[52,53,55]$. In the particular case of the stress-energy tensor this gives

$$
\lim _{\nu \rightarrow i h}\left(\nu^{2}+h^{2}\right) b_{2}(\nu)=\frac{h(2 h-1) \Gamma(2 h+2) C_{\phi_{1} \phi_{1} T_{\mu \nu}} C_{\phi_{3} \phi_{3} T_{\mu \nu}}}{2 \Gamma^{3}(h+1) \Gamma\left(\Delta_{1}-h+1\right) \Gamma\left(\Delta_{3}-h+1\right) \Gamma\left(\Delta_{1}+1\right) \Gamma\left(\Delta_{3}+1\right)} .
$$

Secondly, the OPE coefficients are determined by the Ward identity as in (5.9). This reproduces the relation (5.10) between the bulk gravitational coupling $G_{N}$ and the CFT central charge $C_{T}$.

\subsubsection{Mellin amplitude}

Recalling that given a conformal four-point function, its Mellin amplitude $M(s, t)$ is defined by

$$
\begin{aligned}
& \left\langle\mathcal{O}_{\phi_{1}}\left(P_{1}\right) \mathcal{O}_{\phi_{1}}\left(P_{2}\right) \mathcal{O}_{\phi_{3}}\left(P_{3}\right) \mathcal{O}_{\phi_{3}}\left(P_{4}\right)\right\rangle=\frac{1}{\left(P_{12}\right)^{\Delta_{1}}\left(P_{34}\right)^{\Delta_{3}}} \\
& \quad \times \int \frac{d s d t}{(4 \pi i)^{2}} M(s, t) u^{\frac{t}{2}} v^{-\frac{s+t}{2}} \Gamma\left(\Delta_{1}-\frac{t}{2}\right) \Gamma\left(\Delta_{3}-\frac{t}{2}\right) \Gamma^{2}\left(\frac{-s}{2}\right) \Gamma^{2}\left(\frac{s+t}{2}\right),
\end{aligned}
$$

where the integration contours run parallel to the imaginary axis and the cross-ratios $u$ and $v$ were defined in (6.2). As explained in $[53,55]$, the Mellin amplitude also admits a conformal partial wave expansion,

$$
M(s, t)=\sum_{l=0}^{\infty} \int d \nu b_{l}(\nu) M_{\nu, l}(s, t),
$$

where the partial waves $M_{\nu, l}(s, t)$ involve Mack polynomials and are given in [53] in our conventions.

In the case of the graviton exchange diagram, we find the Mellin amplitude can be expressed as the following integral 


$$
\begin{aligned}
& M(s, t)=\int d \nu\left[P_{\nu, 2}(s, t) b_{2}(\nu)+\frac{(t+2-h)^{2}+\nu^{2}}{\left[\left(h-2 \Delta_{1}\right)^{2}+\nu^{2}\right]\left[\left(h-2 \Delta_{3}\right)^{2}+\nu^{2}\right]} 4 b_{0}(\nu)\right] \\
& \times \frac{\Gamma\left(\frac{2 \Delta_{1}+2-h-i \nu}{2}\right) \Gamma\left(\frac{2 \Delta_{1}+2-h+i \nu}{2}\right) \Gamma\left(\frac{2 \Delta_{3}+2-h-i \nu}{2}\right) \Gamma\left(\frac{2 \Delta_{3}+2-h+i \nu}{2}\right) \Gamma\left(\frac{h-t-i \nu-2}{2}\right) \Gamma\left(\frac{h-t+i \nu-2}{2}\right)}{8 \pi \Gamma\left(\frac{2 \Delta_{1}-t}{2}\right) \Gamma\left(\frac{2 \Delta_{3}-t}{2}\right) \Gamma(i \nu) \Gamma(-i \nu)},
\end{aligned}
$$

where the spin 2 Mack polynomial reads

$$
\begin{aligned}
P_{\nu, 2}(s, t)= & \frac{(2 h-t-3)(2 h-t-1)}{8 h\left[(h-1)^{2}+\nu^{2}\right]} \\
& -\frac{h^{2}-8 h s^{2}-8 h s t-2 h t^{2}-4 h t-2 h+\nu^{2}+t^{2}+4 t+3}{8 h} .
\end{aligned}
$$

The integral over $\nu$ can be done explicitly using the following identities,

$$
\int d \nu \frac{\prod_{\sigma= \pm 1} \prod_{k=1}^{3} \Gamma\left(\frac{a_{k}+\sigma i \nu}{2}\right)}{8 \pi \Gamma(i \nu) \Gamma(-i \nu)}=\Gamma\left(\frac{a_{1}+a_{2}}{2}\right) \Gamma\left(\frac{a_{1}+a_{3}}{2}\right) \Gamma\left(\frac{a_{2}+a_{3}}{2}\right)
$$

and

$$
\frac{1}{\Gamma\left(\frac{a-t}{2}\right) \Gamma\left(\frac{b-t}{2}\right)} \int_{-\infty}^{\infty} \frac{d \nu}{4 \pi} \frac{l(\nu) l(-\nu)}{\left(\nu^{2}+(\Delta-h)^{2}\right)}=\sum_{m=0}^{\infty} \frac{R_{m}}{t-\Delta-2 m}
$$

where

$$
\begin{aligned}
l(\nu) & =\frac{\Gamma\left(\frac{h+i \nu-t}{2}\right) \Gamma\left(\frac{a+i \nu-h}{2}\right) \Gamma\left(\frac{b+i \nu-h}{2}\right)}{\Gamma(i \nu)}, \\
R_{m} & =\Gamma\left(\frac{a+\Delta-2 h}{2}\right) \Gamma\left(\frac{b+\Delta-2 h}{2}\right) \frac{\left(1+\frac{\Delta-a}{2}\right)_{m}\left(1+\frac{\Delta-b}{2}\right)_{m}}{m ! \Gamma(\Delta-h+1+m)} .
\end{aligned}
$$

The final result for generic conformal weights $\Delta_{i}$ of the external scalars and spacetime dimension is

$$
\begin{aligned}
M(s, t)= & C_{\phi_{1} \phi_{1} T_{\mu \nu}} C_{\phi_{3} \phi_{3} T_{\mu \nu}} \sum_{m=0}^{\infty} \frac{\mathcal{Q}_{2, m}(s)}{t-2 h+2-2 m} \\
& +\frac{8 \pi G_{N} \Gamma\left(\Delta_{1}+\Delta_{3}-h\right)\left(h s-\Delta_{1} \Delta_{3}-s\left(\Delta_{1}+\Delta_{3}\right)\right)}{4 \pi^{h} \Gamma\left(\Delta_{1}\right) \Gamma\left(\Delta_{3}\right) \Gamma\left(\Delta_{1}+1-h\right) \Gamma\left(\Delta_{3}+1-h\right)},
\end{aligned}
$$

where

$$
\mathcal{Q}_{2, m}(s)=\frac{(1-2 h) h \Gamma(2 h+2) P_{i h, 2}(s, 2 h-2+2 m)}{4 m ! \Gamma^{4}(h+1)(h+1)_{m} \Gamma\left(\Delta_{1}+1-h-m\right) \Gamma\left(\Delta_{3}+1-h-m\right)},
$$

is the spin 2 Mack polynomial as defined in [53] and the product of OPE coefficients is given by

$$
C_{\phi_{1} \phi_{1} T_{\mu \nu}} C_{\phi_{3} \phi_{3} T_{\mu \nu}}=8 \pi G_{N} \frac{\Delta_{1} \Delta_{3} \Gamma^{3}(h+1)}{\pi^{h} h(2 h-1) \Gamma(2 h+2)} .
$$

Notice that this value for the OPE coefficient is consistent with (6.15) and (6.16). The final result (6.24) for the Mellin amplitude consists of two pieces. The first piece is a sum of poles 
with residues entirely determined by the product of OPE coefficients $C_{\phi_{1} \phi_{1} T_{\mu \nu}} C_{\phi_{3} \phi_{3} T_{\mu \nu}}$. This piece follows from the structure of the Mellin amplitudes and was known before (see appendix A.3 of [53]). The second term in (6.24) was not known before and required a careful treatment of the contact terms in the graviton propagator.

In the large $s \sim t$ limit, the Mellin amplitude simplifies to

$$
M(s, t) \approx-\frac{8 \pi G_{N} \Gamma\left(\Delta_{1}+\Delta_{3}-h+1\right)}{4 \pi^{h} \Gamma\left(\Delta_{1}\right) \Gamma\left(\Delta_{3}\right) \Gamma\left(\Delta_{1}+1-h\right) \Gamma\left(\Delta_{3}+1-h\right)} \frac{s^{2}+s t}{t} .
$$

Using the flat space limit of AdS [15], this corresponds to the following bulk scattering amplitude between massless scalars ${ }^{7}$

$$
\mathcal{T}(S, T) \approx-8 \pi G_{N} \frac{S^{2}+S T}{T}
$$

where $S$ and $T$ are the usual flat space Mandelstam invariants. This is the correct result for the graviton exchange amplitude between minimally coupled massless scalars $[56,57]$. Another check of the result (6.24) is the property $M(s, t)=M(-s-t, t)$, that follows from the invariance of the Witten diagram under the exchange of points $1 \leftrightarrow 2$ (or $3 \leftrightarrow 4$ ). Finally, as an example, we present the result when $\Delta_{1}=\Delta_{3}=2 h=4$,

$$
M(s, t)=-\frac{2 G_{N}}{3 \pi}\left(\frac{(s+4)(s+2)}{(t-6)}+\frac{8(s+2)^{2}}{(t-4)}+\frac{6 s(s+2)+8}{(t-2)}+5(3 s+8)\right),
$$

which matches the result obtained in [15] (after the appropriate change of conventions).

The four-point function associated to an AdS graviton exchange between scalar primary operators can be expanded in conformal blocks in the crossed channel. More precisely, we can write

$$
\left\langle\mathcal{O}_{\phi_{1}}\left(P_{1}\right) \mathcal{O}_{\phi_{1}}\left(P_{2}\right) \mathcal{O}_{\phi_{3}}\left(P_{3}\right) \mathcal{O}_{\phi_{3}}\left(P_{4}\right)\right\rangle=\sum_{n, l=0}^{\infty} p(n, l) G_{\Delta(n, l), l}^{(13)(24)}\left(P_{1}, \ldots, P_{4}\right),
$$

where $G_{\Delta(n, l), l}^{(13)(24)}$ is the conformal block describing the exchange of an operator with spin $l$ and dimension

$$
\Delta(n, l)=\Delta_{1}+\Delta_{3}+2 n+l+\gamma(n, l),
$$

in the $(13)(24)$ channel. The anomalous dimensions $\gamma(n, l)$ are small if the interactions in the dual AdS space are weak. In fact, we can think of $\gamma(n, l)$ as the gravitational binding energy of a two particle state with angular momentum $l$ [58]. The quantum number $n$ increases the ellipticity of the corresponding classical orbits and we will set it to zero for simplicity. Using the techniques described in [53], one can compute the anomalous dimensions in terms of the Mellin amplitude. To first order in the gravitational coupling we obtain

$$
\begin{array}{r}
\gamma(0, l)=-\int_{-i \infty}^{i \infty} \frac{d t}{2 \pi i} M(0, t) \Gamma^{2}\left(\frac{t}{2}\right) \Gamma\left(\Delta_{1}-\frac{t}{2}\right) \Gamma\left(\Delta_{3}-\frac{t}{2}\right) \\
\times{ }_{3} F_{2}\left(-l, l+\Delta_{1}+\Delta_{3}-1, \frac{t}{2} ; \Delta_{1}, \Delta_{3} ; 1\right),
\end{array}
$$

\footnotetext{
${ }^{7}$ See equation (132) of [53] for the flat space limit formula in the present conventions.
} 
where the Mellin amplitude was given in (6.24). Using the large $l$ asymptotic behaviour,

$$
{ }_{3} F_{2}\left(-l, l+\Delta_{1}+\Delta_{3}-1, \frac{t}{2} ; \Delta_{1}, \Delta_{3} ; 1\right) \approx \frac{\Gamma\left(\Delta_{1}\right) \Gamma\left(\Delta_{3}\right)}{\Gamma\left(\Delta_{1}-\frac{t}{2}\right) \Gamma\left(\Delta_{3}-\frac{t}{2}\right)} \frac{1}{l^{t}},
$$

we conclude that the large spin behaviour of the anomalous dimension $\gamma(0, l)$ is controlled the leading $t$-pole of the Mellin amplitude (6.24). This gives

$$
\gamma(0, l) \approx-C_{\phi_{1} \phi_{1} T_{\mu \nu}} C_{\phi_{3} \phi_{3} T_{\mu \nu}} \frac{\Gamma(2 h+2) \Gamma\left(\Delta_{1}\right) \Gamma\left(\Delta_{3}\right)}{2 \Gamma^{2}(h+1) \Gamma\left(\Delta_{1}-h+1\right) \Gamma\left(\Delta_{3}-h+1\right)} \frac{1}{l^{2 h-2}},
$$

which agrees with the results of [59-61] (in particular see formula (B.33) of [60]), if we assume that the stress-energy tensor is the operator with minimal twist.

\section{Concluding remarks}

In this work we developed the embedding formalism to deal with tensor fields in Anti-de Sitter spacetime. In particular, we encoded symmetric traceless tensors into polynomials of an auxiliary null vector and found differential operators that implement the laplacian and divergence in this language. With this technology, we were able to obtain the bulk-to-bulk propagator of massive spinning particles in AdS. We also found a split representation for these propagators. This is a very useful integral representation because it is based on the product of bulk-to-boundary propagators. For example, it leads directly to the conformal partial wave expansion of four-point Witten diagrams. Up to spin 2, we gave complete split representations of the bulk-to-bulk propagator, including contact terms. By a careful study of the massless limit, we obtained the split representation of the graviton propagator which was the subject of some controversy in the literature [23, 24]. For spin greater than 2 , we only gave the split representation up to contact terms, i.e. for non-coincident points. It should also be possible to obtain the complete split representation, for instance studying the non-local equations of motion proposed in [34].

We illustrated the use of the embedding formalism and the split representation of the propagators, by computing three and four-point functions involving tensor fields. In particular, we obtained a closed formula for the conformal partial wave expansion and the Mellin amplitude associated to graviton exchange between to scalars of arbitrary conformal weight in general spacetime dimension.

There are several natural extensions of our work. An obvious one is to study antisymmetric and mixed symmetry tensors. More interesting, would be the generalization to spinorial fields. Another simple extension is the study of higher spin fields in de Sitter space. We leave these ideas for the future, hoping to have convinced the reader that embedding methods can be very powerful in the treatment of higher spin fields in AdS, for example in the computation of Witten diagrams.

\section{Acknowledgments}

We are grateful to Perimeter Institute for the great hospitality in the summer of 2012 where this work was initiated. M.S.C and V.G also thank IPMU at Tokyo University for 
the great hospitality during the progress of this work. J.P. wishes to thank the hospitality of KITP at UCSB where part of this work was developed. The research leading to these results has received funding from the [European Union] Seventh Framework Programme under grant agreements No 269217 and No 317089. This work was partially funded by the grant CERN/FP/123599/2011 and by the Matsumae International Foundation in Japan. Centro de Fisica do Porto is partially funded by the Foundation for Science and Technology of Portugal (FCT). The work of V.G. is supported by the FCT fellowship SFRH/BD/68313/2010.

\section{A Harmonic functions in flat space}

In this appendix we collect some basic facts about harmonic functions in flat space, hopefully this will make the transition to AdS more transparent. The equation for the propagator of a spin $J$ field in flat space is simpler than the AdS one (3.13),

$$
\left(\partial^{2}-m^{2}\right) \Pi_{m}^{A_{1} \ldots A_{J} B_{1} \ldots B_{J}}(X-\bar{X})=-\mathcal{P}_{m^{2}}^{A_{1} \ldots A_{J} B_{1} \ldots B_{J}} \delta(X-\bar{X}),
$$

where $\mathcal{P}_{m^{2}}$ is a projector. For example,

$$
\mathcal{P}_{m^{2}}^{A B}=\eta^{A B}-\frac{\partial^{A} \partial^{B}}{m^{2}}
$$

More generally, the projector is

$$
\mathcal{P}_{m^{2}}^{A_{1} \ldots A_{J} B_{1} \ldots B_{J}}=\frac{1}{J !} \sum_{\text {perm } B_{i}} \prod_{i=1}^{J} \mathcal{P}_{m^{2}}^{A_{i} B_{i}}-\left(A_{i} \text { and } B_{i} \text { traces }\right)
$$

The projector commutes with the Laplacian, therefore

$$
\Pi_{m}^{A_{1} \ldots A_{J} B_{1} \ldots B_{J}}(X-\bar{X})=\mathcal{P}_{m^{2}}^{A_{1} \ldots A_{J} B_{1} \ldots B_{J}} \Pi_{m}(X-\bar{X}) .
$$

The analogue of AdS harmonic function (4.3) in flat space is

$$
\begin{aligned}
\Omega_{\nu}^{A_{1} \ldots A_{J} B_{1} \ldots B_{J}}(X-\bar{X}) & =\frac{i \nu}{2 \pi}\left[\Pi_{i \nu}^{A_{1} \ldots A_{J} B_{1} \ldots B_{J}}(X-\bar{X})-\Pi_{-i \nu}^{A_{1} \ldots A_{J} B_{1} \ldots B_{J}}(X-\bar{X})\right] \\
& =\mathcal{P}_{-\nu^{2}}^{A_{1} \ldots A_{J} B_{1} \ldots B_{J}} \Omega_{\nu}(X-\bar{X}) .
\end{aligned}
$$

The harmonic function can be written using an integral representation

$$
\Omega_{\nu}(X)=\nu \int d K e^{i K \cdot X} \delta\left(K^{2}-\nu^{2}\right)
$$

that can be explicitly checked from

$$
\Pi_{ \pm i \nu}(X)=\int d K \frac{e^{i K \cdot X}}{K^{2}+( \pm i \nu+\epsilon)^{2}}=\int d K \frac{e^{i K \cdot X}}{K^{2}-\nu^{2} \pm i \epsilon} .
$$

The generalization of harmonic functions $\Omega_{\nu}$ to spin $J$ is now straightforward,

$$
\Omega_{\nu}^{A_{1} \ldots A_{J} B_{1} \ldots B_{J}}(X)=\nu \int d K e^{i K \cdot X} \delta\left(K^{2}-\nu^{2}\right) \frac{1}{J !} \sum_{\pi_{B_{i}}} \prod_{i=1}^{J}\left(\eta^{A_{i} B_{i}}-\frac{K^{A_{i}} K^{B_{i}}}{\nu^{2}}\right)-\text { traces }
$$


where the sum is over the permutations of all $B_{i}$ indices. For example, for spin 2 we have

$$
\begin{aligned}
\Omega_{\nu}^{A_{1} A_{2} B_{1} B_{2}}(X)= & \frac{\nu}{2} \int d K e^{i K \cdot X} \delta\left(K^{2}-\nu^{2}\right) \\
& {\left[\left(\eta^{A_{1} B_{1}}-\frac{K^{A_{1}} K^{B_{1}}}{\nu^{2}}\right)\left(\eta^{A_{2} B_{2}}-\frac{K^{A_{2}} K^{B_{2}}}{\nu^{2}}\right)\right.} \\
& +\left(\eta^{A_{1} B_{2}}-\frac{K^{A_{1}} K^{B_{2}}}{\nu^{2}}\right)\left(\eta^{A_{2} B_{1}}-\frac{K^{A_{2}} K^{B_{1}}}{\nu^{2}}\right) \\
& \left.-\frac{2}{d}\left(\eta^{A_{1} A_{2}}-\frac{K^{A_{1}} K^{A_{2}}}{\nu^{2}}\right)\left(\eta^{B_{1} B_{2}}-\frac{K^{B_{1}} K^{B_{2}}}{\nu^{2}}\right)\right],
\end{aligned}
$$

with $d+1$ being the spacetime dimension.

These harmonic functions satisfy orthogonality and completeness relations similar to (4.7) and (4.11). For scalars, we have

$$
\int d \nu \Omega_{\nu}(X-\bar{X})=\delta(X-\bar{X})
$$

and

$$
\int d Y \Omega_{\nu}(X-Y) \Omega_{\bar{\nu}}(Y-\bar{X})=\frac{\delta(\nu+\bar{\nu})+\delta(\nu+\bar{\nu})}{2} \Omega_{\nu}(X-\bar{X}) .
$$

The generalization of (A.11) to non-zero spin is simply

$$
\begin{aligned}
& \int d Y \Omega_{\nu}^{A_{1} \ldots A_{J} B_{1} \ldots B_{J}}(X-Y) \Omega_{\bar{\nu}}^{B_{1} \ldots B_{J} C_{1} \ldots C_{J}}(Y-\bar{X}) \\
= & \frac{\delta(\nu+\bar{\nu})+\delta(\nu+\bar{\nu})}{2} \Omega_{\nu}^{A_{1} \ldots A_{J} C_{1} \ldots C_{J}}(X-\bar{X}) .
\end{aligned}
$$

On the other hand, the generalization of (A.10) to generic spin is more subtle. For spin 1 and 2 one has

$$
\int d \nu\left[\Omega_{\nu}^{A B}(X-\bar{X})+\frac{1}{\nu^{2}} \partial^{A} \bar{\partial}^{B} \Omega_{\nu}(X-\bar{X})\right]=\eta^{A B} \delta(X-\bar{X}),
$$

and

$$
\begin{aligned}
\int d \nu & {\left[\Omega_{\nu}^{A_{1} A_{2} B_{1} B_{2}}(X-\bar{X})+\frac{1}{2 \nu^{2}} \partial^{A_{1}} \bar{\partial}^{B_{1}} \Omega_{\nu}^{A_{2} B_{2}}(X-\bar{X})+\frac{1}{2 \nu^{2}} \partial^{A_{2}} \bar{\partial}^{B_{2}} \Omega_{\nu}^{A_{1} B_{1}}(X-\bar{X})\right.} \\
& +\frac{1}{2 \nu^{2}} \partial^{A_{1}} \bar{\partial}^{B_{2}} \Omega_{\nu}^{A_{2} B_{1}}(X-\bar{X})+\frac{1}{2 \nu^{2}} \partial^{A_{2}} \bar{\partial}^{B_{1}} \Omega_{\nu}^{A_{1} B_{2}}(X-\bar{X}) \\
& \left.+\frac{d+1}{d \nu^{4}} \partial^{A_{1}} \partial^{A_{2}} \bar{\partial}^{B_{1}} \bar{\partial}^{B_{2}} \Omega_{\nu}(X-\bar{X})+\frac{1}{d} \eta^{A_{1} A_{2}} \eta^{B_{1} B_{2}} \Omega_{\nu}(X-\bar{X})\right] \\
= & \frac{1}{2}\left(\eta^{A_{1} B_{1}} \eta^{A_{2} B_{2}}+\eta^{A_{1} B_{2}} \eta^{A_{2} B_{1}}\right) \delta(X-\bar{X}),
\end{aligned}
$$

respectively. This should be compared with the completeness formula (4.11) for harmonic functions in AdS. In fact, the limit $\nu \gg 1$ of (4.11) matches exactly the flat space formulas above. 


\section{B Embedding space operations}

The embedding formalism gives a compact form to write all tensor structures of the spin $J$ propagators in AdS and it allows simpler tensor manipulations. The main aim of this section is to compute the trace over all structures present in the propagator,

$$
\left(K_{1} \cdot K_{2}\right)^{J}\left(W_{12}\right)^{J-l}\left(\left(W_{1} \cdot X_{2}\right)\left(W_{2} \cdot X_{1}\right)\right)^{l}
$$

To make the derivation more pedagogical, we will present two intermediate tensor operations that are necessary to compute the trace (B.1).

First operation - symmetric traceless contraction. The first operation is the action of the differential operator $K_{1}$ on the $W_{1}$ variables. ${ }^{8}$ For that we will use the identity,

$$
\frac{(K \cdot P)^{J}(W \cdot Q)^{J}}{J !\left(h-\frac{1}{2}\right)_{J}}=B^{\left\{A_{1}\right.} \ldots B^{\left.A_{J}\right\}} G_{\left\{A_{1}\right.} \ldots G_{\left.A_{J}\right\}}=\frac{J !\left(B^{2} G^{2}\right)^{\frac{J}{2}}}{2^{J}\left(h-\frac{1}{2}\right)_{J}} C_{J}^{h-\frac{1}{2}}(t),
$$

where $C_{J}^{h-\frac{1}{2}}(t)$ is the Gegenbauer polynomial and

$$
B_{A}=P_{A}+(X \cdot P) X_{A}, \quad G_{A}=Q_{A}+(Q \cdot X) X_{A}, \quad t=\frac{P \cdot Q+(P \cdot X)(Q \cdot X)}{(P \cdot X)\left((Q \cdot X)^{2}+Q^{2}\right)^{\frac{1}{2}}}
$$

where it was used that $P^{2}=0$. This last expression will also be useful to compute (E.3) below.

Second operation. The second operation is the action of the operator $K$ on a specific vector containing the polarization vector $W$,

$$
K_{A_{1}} \ldots K_{A_{J}}\left(W_{A_{1}}+\alpha Y_{A_{1}}(W \cdot Y)\right) \ldots\left(W_{A_{J}}+\alpha Y_{A_{J}}(W \cdot Y)\right)
$$

where $\alpha$ is any function that does not depend on $W$, and $Y$ is a generic bulk vector satisfying $Y^{2}=-1$. The result of this operation is

$$
(2 h-1)_{J}\left(h+\frac{1}{2}\right)_{J}{ }_{2} F_{1}\left(1,-J, h+\frac{1}{2}, \frac{\alpha(1+X \cdot Y)(1-X \cdot Y)}{2}\right) .
$$

This expression was guessed by performing the operation for $J=1, \ldots, 5$ and should be valid for any $J$.

\footnotetext{
${ }^{8}$ In this process we consider $K_{2}$ as a generic vector. To compute the trace (B.1), $K_{2}$ needs to act on the variables $W_{2}$ and so we have to remember that the differential operator $K_{2}$ has to be placed to the left of all vectors $W_{2}$.
} 
Trace over structures of propagator. Multiplying (B.1) by $\frac{J ! x^{l}}{l !(J-l) !}$ and summing over $l$ gives a generator of all the structures,

$$
\left(K_{1} \cdot K_{2}\right)^{J}\left(W_{1} \cdot\left(W_{2}+x\left(W_{2} \cdot X_{1}\right) X_{2}\right)\right)^{J} .
$$

The original expression can be recovered expanding the final result in $x$. Applying (B.2) to this expression, we obtain

$$
\sum_{k=0}^{|J / 2|} \frac{\theta^{k}(J !)^{2}\left(h-\frac{1}{2}\right)_{J-k}}{2^{2 k} k !(J-2 k) !}\left(K_{2} \cdot X_{1}\right)^{2 k}\left(K_{2} \cdot\left(W_{2}+\alpha X_{1}\left(W_{2} \cdot X_{1}\right)\right)\right)^{J-2 k}\left(W_{2} \cdot X_{1}\right)^{2 k},
$$

where $|J / 2|$ is the integer part of $J / 2, \alpha=1+x\left(X_{1} \cdot X_{2}\right)$ and $\theta=x^{2}-\alpha^{2}$. In this calculation we have used $K_{2} \cdot\left(x X_{2}\left(X_{1} \cdot W_{2}\right)\right)=0$. The evaluation of the expression outside the fraction in (B.7) can be done by multiplying by $2 \frac{J !}{(2 k) !(J-2 k) !} y^{2 k}$ and summing over $k$, giving

$$
\left(K_{2} \cdot\left(W_{2}+(\alpha+y) X_{1}\left(W_{2} \cdot X_{1}\right)\right)\right)^{J}+\left(K_{2} \cdot\left(W_{2}+(\alpha-y) X_{1}\left(W_{2} \cdot X_{1}\right)\right)\right)^{J} .
$$

Then, using the second tensor operation (B.4) we obtain

$$
2(2 h-1)_{J}\left(h+\frac{1}{2}\right) \sum_{J} \sum_{m=0}^{J} \sum_{k=0}^{|m / 2|} \frac{(-J)_{m}(-1)^{m}}{2^{m}\left(h+\frac{1}{2}\right)_{m}} \frac{m !(u(2+u))^{m} \alpha^{m-2 k} y^{2 k}}{(m-2 k) !(2 k) !} .
$$

Notice that, as expected, this expression just depends on even powers of $y$. Thus, the expression outside the fraction in (B.7) is recovered by matching powers of $y$,

$$
\frac{(2 h-1)_{J}\left(h+\frac{1}{2}\right)_{J}}{J !} \sum_{m=0}^{J} \frac{(-J)_{m}(-1)^{m}}{2^{m}\left(h+\frac{1}{2}\right)_{m}} \frac{m !(u(2+u))^{m} \alpha^{m-2 k}}{(1+J-2 k)_{m-J}} .
$$

Joining all pieces in a single expression we conclude that

$$
\begin{aligned}
& \frac{1}{J !(2 h-1)_{J}\left(h+\frac{1}{2}\right)_{J}}\left(K_{1} \cdot K_{2}\right)^{J}\left(W_{12}+x\left(W_{1} \cdot X_{2}\right)\left(W_{2} \cdot X_{1}\right)\right)^{J} \\
= & \sum_{k=0}^{|J / 2|} \sum_{m=0}^{J} \frac{\left(h-\frac{1}{2}\right)_{J-k}}{2^{2 k} k !} \frac{(-J)_{m} m !(u(2+u))^{m}\left(2(1+u) x-1-u(2+u) x^{2}\right)^{k}(x(1+x)-1)^{m-2 k}}{2^{m}\left(h+\frac{1}{2}\right)_{m}(m-2 k) !} .
\end{aligned}
$$

This simplifies dramatically in the limit $u \rightarrow 0$, as only the $x=0$ term survives. The final result is

$$
\left(K_{1} \cdot K_{2}\right)^{J}\left(W_{12}+x\left(W_{1} \cdot X_{2}\right)\left(W_{2} \cdot X_{1}\right)\right)^{J}=(2 h-1)_{J}\left(h+\frac{1}{2}\right)_{J}\left(h-\frac{1}{2}\right)_{J} J !+O(u) .
$$

\section{Split representation of the bulk-to-bulk propagator}

The harmonic functions $\Omega_{\nu, J}$ can be defined by the integral over the boundary point that connects two bulk-to-boudary propagators, as in equation (4.1). Alternatively, the harmonic functions $\Omega_{\nu, J}$ can be defined by the difference of two bulk-to-bulk propagators with 
dimensions $h+i \nu$ and $h-i \nu$, as in equation (4.3). The goal of this appendix is to show that these are equivalent definitions.

We start from equation (4.1),

$$
\frac{\nu^{2}}{\pi J !(h-1)_{J}} \int_{\partial} d P \Pi_{h+i \nu, J}\left(X_{1}, P ; W_{1}, D_{Z}\right) \Pi_{h-i \nu, J}\left(X_{2}, P ; W_{2}, Z\right) .
$$

The boundary contraction can be done using an identity similar to (B.2) [11, 13], with result

$$
\frac{2^{J} J ! \mathcal{C}_{h+i \nu, J} \mathcal{C}_{h-i \nu, J} \nu^{2}}{\pi(h-1)_{J}} \int_{\partial} d P \frac{\left(\left(P \cdot W_{1}\right)\left(P \cdot W_{2}\right)\right)^{J} C_{J}^{h-1}(t)}{\left(-2 P \cdot X_{1}\right)^{h+i \nu+J}\left(-2 P \cdot X_{2}\right)^{h-i \nu+J}},
$$

where $t$ is defined as

$$
t=X_{1} \cdot X_{2}+\frac{\left(P \cdot X_{1}\right)\left(P \cdot X_{2}\right)}{\left(P \cdot W_{1}\right)\left(P \cdot W_{2}\right)} W_{1} \cdot W_{2}-\frac{P \cdot X_{1}}{P \cdot W_{1}} W_{1} \cdot X_{2}-\frac{P \cdot X_{2}}{P \cdot W_{2}} W_{2} \cdot X_{1} .
$$

It is possible to choose polarizations such that $W_{1} \cdot X_{2}$ and $W_{2} \cdot X_{1}$ vanish. With this specific choice only the term in $\left(W_{12}\right)^{J}$ survives. Using the definition of the Gegenbauer polynomial and performing a Feynman parametrization, (C.2) becomes

$$
\begin{aligned}
& \frac{J ! \mathcal{C}_{h+i \nu, J} \mathcal{C}_{h-i \nu, J} \nu^{2}}{\pi(h-1)_{J}} \sum_{l=0}^{J} \sum_{k=0}^{|l / 2|} \int_{\partial} d P \int_{0}^{\infty} \frac{d q}{q} \\
& \times \frac{(-1)^{k+l} 2^{l} q^{h+l} \Gamma(2 h+2 l)(h-1)_{J-k}\left(W_{12}\right)^{J-l}\left(\left(W_{1} \cdot P\right)\left(W_{2} \cdot P\right)\right)^{l}\left(2 X_{1} \cdot X_{2}\right)^{l-2 k}}{k !(l-2 k) !(J-l) ! q^{i \nu} \Gamma(h+i \nu+l) \Gamma(h-i \nu+l)(-2 P \cdot Y)^{2 h+2 l}},
\end{aligned}
$$

with $Y=X_{1}+q X_{2}$. The integral over the boundary point $P$ is conformal and can be done using the equality [14]

$$
\int_{\partial} d P \frac{P^{A_{1}} \ldots P^{A_{2 l}}}{(-2 P \cdot Y)^{2 h+2 l}}=\frac{\pi^{h}(2 h+2 l)_{-h} Y^{A_{1}} \ldots Y^{A_{2 l}}}{\left(-Y^{2}\right)^{h+2 l}}-\text { traces } .
$$

As the integration variables are contracted with $W_{1}$ and $W_{2}$, and since $W_{i} \cdot X_{j}=0$, we have

$$
\int_{\partial} d P \frac{\left(W_{1} \cdot P\right)^{l}\left(W_{2} \cdot P\right)^{l}}{(-2 P \cdot Y)^{2 h+2 l}}=\frac{\pi^{h}(2 h+2 l)_{-h} l !}{(-2)^{l}(h+l)_{l}\left(-Y^{2}\right)^{h+l}}\left(W_{12}\right)^{l} .
$$

The sum over $k$ can be done and gives an hypergeometric function, so (C.4) becomes

$$
\frac{\mathcal{C}_{h+i \nu, J} \mathcal{C}_{h-i \nu, J} \nu^{2}}{\pi} \sum_{l=0}^{J} \int d q \frac{\pi^{h} J ! \Gamma(h+l)\left(W_{12}\right)^{J}{ }_{2} F_{1}\left(-l, \frac{3}{2}-h-J, 3-2 h-2 J,-\frac{2}{u}\right)}{(J-l) ! \Gamma(h+i \nu+l) \Gamma(h-i \nu+l)(-2 u)^{-l} q^{1-h+i \nu-l}\left(-Y^{2}\right)^{h+l}} .
$$

Finally, using the equality

$$
\begin{aligned}
\int_{0}^{\infty} \frac{d \alpha}{\alpha} \frac{\alpha^{-c}}{\left(\frac{(1+\alpha)^{2}}{\alpha}+2 u\right)^{b}}= & \frac{\Gamma(b+c) \Gamma(-c)}{\Gamma(b)(2 u)^{b+c}}{ }_{2} F_{1}\left(\frac{1}{2}+c, b+c, 1+2 c,-\frac{2}{u}\right) \\
& +\frac{\Gamma(b-c) \Gamma(c)}{\Gamma(b)(2 u)^{b-c}}{ }_{2} F_{1}\left(\frac{1}{2}-c, b-c, 1-2 c,-\frac{2}{u}\right),
\end{aligned}
$$



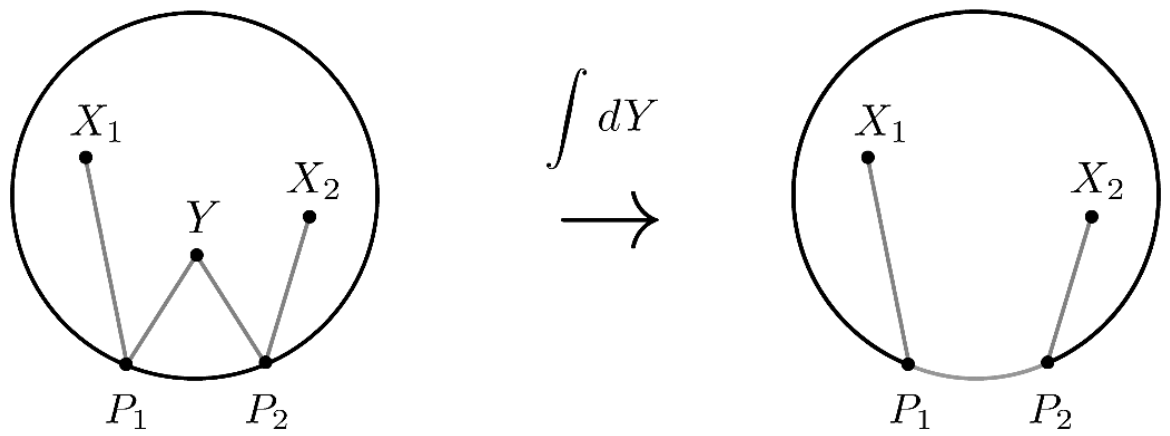

Figure 6. Product of two harmonic functions $\Omega_{\nu, J}\left(X_{1}, Y ; W_{1}, K\right)$ and $\Omega_{\bar{\nu}, J}\left(Y, X_{2} ; W, W_{2}\right)$, where $K$ acts on the polarisation vector $W$ of $Y$, represented as the integral over bulk-to-boudary propagators. After integration over the bulk point $Y$, we are left with two bulk-to-boudary propagators and one two-point function on the boundary.

the integral over $q$ can also be expressed in terms of hypergeometric functions. These two hypergeometric functions correspond precisely to the two propagators with $\Delta=h \pm i \nu$ in (4.3). Thus, from the term proportional to $\left(W_{12}\right)^{J}$ in the propagator (3.7), we obtain

$$
\begin{aligned}
& g_{0}(u)=\frac{2 \pi^{h} J ! \mathcal{C}_{\Delta, J} \mathcal{C}_{2 h-\Delta, J} \Gamma(1+h-\Delta)}{\Gamma(2 h-\Delta)(2 u)^{\Delta}} \\
& \times \sum_{l=0}^{J} \frac{(-1)^{l}{ }_{2} F_{1}\left(-l, \frac{3}{2}-h-J, 3-2 h-2 J,-\frac{2}{u}\right){ }_{2} F_{1}\left(\frac{1}{2}+\Delta-h, \Delta+l, 1+2 \Delta-2 h,-\frac{2}{u}\right)}{(J-l) !(2 h-\Delta)_{l}} .
\end{aligned}
$$

We checked up to $J=5$ that this expression for $g_{0}$ is reproduced by formula (3.12) where the functions $f_{i}^{(i+k)}=h_{i}^{(k)}$ are determined by (3.20) and (3.22). This shows that (4.3) is equivalent to (4.1).

\section{AdS harmonic functions}

Orthogonality. The AdS harmonic functions satisfy the orthogonality relation (4.7) that says that the integral of two $\Omega$ functions over a commom bulk point gives again an $\Omega$ function. The argument that led to the orthogonality relation could not fix the overall constant multiplying the right hand side of (4.7). The goal of this appendix is to fix this constant by evaluating this bulk integral using the representation (4.1) of the AdS harmonic functions. This computation is represented in figure 6 . We need to do the following integral

$$
\begin{aligned}
\frac{1}{J !\left(h-\frac{1}{2}\right)_{J}}\left(\frac{\nu \bar{\nu}}{\pi J !(h-1)_{J}}\right)^{2} \int & d P_{1} d P_{2} d Y \Pi_{h+i \nu, J}\left(X_{1}, P_{1} ; W_{1}, D_{Z_{1}}\right) \Pi_{h-i \nu, J}\left(Y, P_{1} ; K, Z_{1}\right) \\
& \left.\times \Pi_{h-i \bar{\nu}, J}\left(Y, P_{2} ; W, D_{Z_{2}}\right) \Pi_{h+i \bar{\nu}, J}\left(X_{2}, P_{2} ; W_{2}, Z_{2}\right), \quad \text { (D. } 1\right)
\end{aligned}
$$

where the operator $K$ and $D_{Z}$ were included to perform the index contraction between the propagators. We start by performing the integral over the bulk point $Y$,

$$
\frac{1}{J !\left(h-\frac{1}{2}\right)_{J}} \int d Y \Pi_{h-i \nu, J}\left(Y, P_{1} ; K, Z_{1}\right) \Pi_{h-i \bar{\nu}, J}\left(Y, P_{2} ; W, Z_{2}\right) .
$$


After the contraction over the polarizations, this gives ${ }^{9}$

$$
(-4)^{J} \mathcal{C}_{h-i \nu, J} \mathcal{C}_{h-i \bar{\nu}, J} \int d Y I_{\nu, \bar{\nu}, J},
$$

where

$$
\begin{aligned}
& I_{\nu, \bar{\nu}, J}=\frac{1}{\left(-2 P_{1} \cdot Y\right)^{h-i \nu+J}\left(-2 P_{2} \cdot Y\right)^{h-i \bar{\nu}+J}}\left(\left(P_{1} \cdot Z_{2}\right)\left(P_{2} \cdot Y\right)\left(Y \cdot Z_{1}\right)\right. \\
& \left.+\left(P_{2} \cdot Z_{1}\right)\left(P_{1} \cdot Y\right)\left(Y \cdot Z_{2}\right)-\left(P_{1} \cdot P_{2}\right)\left(Y \cdot Z_{1}\right)\left(Y \cdot Z_{2}\right)-\left(P_{1} \cdot Y\right)\left(P_{2} \cdot Y\right)\left(Z_{1} \cdot Z_{2}\right)\right)^{J}
\end{aligned}
$$

and the constant $\mathcal{C}_{\Delta, J}$ is defined in (3.46). Next we define the differential operator

$$
\begin{aligned}
\mathcal{D}= & D_{21} D_{12}+D_{12} D_{21}-H_{12}\left(\left(P_{1} \cdot \partial_{P_{1}}\right)\left(P_{2} \cdot \partial_{P_{2}}\right)+\frac{1}{2}\left(P_{1} \cdot \partial_{P_{1}}+P_{2} \cdot \partial_{P_{2}}\right)\right. \\
& \left.-2\left(Z_{1} \cdot \partial_{Z_{1}}\right)\left(P_{1} \cdot \partial_{P_{1}}+P_{2} \cdot \partial_{P_{2}}\right)-2 Z_{1} \cdot \partial_{Z_{1}}+4\left(Z_{1} \cdot \partial_{Z_{1}}\right)\left(Z_{1} \cdot \partial_{Z_{1}}\right)\right),
\end{aligned}
$$

where

$$
\begin{aligned}
H_{12} & =2\left(\left(P_{1} \cdot Z_{2}\right)\left(P_{2} \cdot Z_{1}\right)-\left(P_{1} \cdot P_{2}\right)\left(Z_{1} \cdot Z_{2}\right)\right) \\
D_{i j} & =\left(Z_{j} \cdot P_{i}\right)\left(Z_{j} \cdot \frac{\partial}{\partial Z_{j}}-P_{j} \cdot \frac{\partial}{\partial P_{j}}\right)+\left(P_{j} \cdot P_{i}\right)\left(Z_{j} \cdot \frac{\partial}{\partial P_{j}}\right) .
\end{aligned}
$$

Then we have

$$
\frac{1}{4(h-i \nu+J)(h-i \bar{\nu}+J)} \mathcal{D}\left(\left(P_{12}\right)^{J} I_{\nu, \bar{\nu}, J}\right)=\left(P_{12}\right)^{J+1} I_{\nu, \bar{\nu}, J+1} .
$$

Thus, the differential operator $\mathcal{D}$ allows us to write

$$
I_{\nu, \bar{\nu}, J}=\frac{1}{4^{J}(h-i \nu)_{J}(h-i \bar{\nu})_{J}\left(P_{12}\right)^{J}} \mathcal{D}^{J} \frac{1}{\left(-2 P_{1} \cdot Y\right)^{h-i \nu}\left(-2 P_{2} \cdot Y\right)^{h-i \bar{\nu}}} .
$$

So the integral (D.3) can be rewritten as

$$
\frac{\mathcal{C}_{h-i \nu, J} \mathcal{C}_{h-i \bar{\nu}, J}}{(h-i \nu)_{J}(h-i \bar{\nu})_{J}\left(P_{12}\right)^{J}} \mathcal{D}^{J} \int d Y \frac{1}{\left(-2 P_{1} \cdot Y\right)^{h-i \nu}\left(-2 P_{2} \cdot Y\right)^{h-i \bar{\nu}}} .
$$

Now the integral over $Y$ only involves scalar bulk-to-boudary propagators. So, let us analyze

$$
\begin{aligned}
& \int \frac{d Y}{\left(-2 P_{1} \cdot Y\right)^{h-i \nu}\left(-2 P_{2} \cdot Y\right)^{h-i \bar{\nu}}} \\
= & \int d^{d} x \int_{0}^{\infty} \frac{d z}{z^{d+1}} \frac{z^{2 h-i \nu-i \bar{\nu}+\epsilon}}{\left[z^{2}+\left(x-x_{1}\right)^{2}\right]^{h-i \nu}\left[z^{2}+\left(x-x_{2}\right)^{2}\right]^{h-i \bar{\nu}}},
\end{aligned}
$$

where the bulk-to-boudary propagator was written in Poincaré coordinates and we have introduced a regulator $z^{\epsilon}$, planning to take the limit $\epsilon \rightarrow 0$ at the end. Using the Fourier representation of the propagator, we obtain

$$
\int_{0}^{\infty} \frac{d z}{z} \int \frac{d^{d} k}{(2 \pi)^{d}} z^{-i \nu-i \bar{\nu}+\epsilon} e^{i k \cdot\left(x_{1}-x_{2}\right)} \widehat{\Pi}_{i \nu}(|k| z) \widehat{\Pi}_{i \bar{\nu}}(|k| z)
$$

\footnotetext{
${ }^{9}$ Notice that generally contraction of symmetric and traceless structures gives a Gegenbauer polynomial. In this particular case the expression is simplified because the denominator of $t$ in (B.2) vanishes.
} 
where the function $\widehat{\Pi}_{i \nu}(|k| z)$ is just the Fourier transform

$$
\begin{aligned}
& \int d^{d} x \frac{e^{i k \cdot x}}{\left(z^{2}+x^{2}\right)^{h-i \nu}} \\
= & \int_{0}^{\infty} \frac{d s}{s} s^{h-i \nu} \frac{e^{-s z^{2}} e^{-\frac{k^{2}}{4 s}}}{\Gamma(h-i \nu)} \int d^{d} x e^{-s\left(x-i \frac{k}{2 s}\right)^{2}}=\frac{\pi^{h}}{\Gamma(h-i \nu)} \int_{0}^{\infty} \frac{d s}{s} s^{-i \nu} e^{-s z^{2}-\frac{k^{2}}{4 s}} .
\end{aligned}
$$

This turns (D.12) into

$$
\frac{\pi^{2 h}}{\Gamma(h-i \nu) \Gamma(h-i \bar{\nu})} \int \frac{d^{d} k}{(2 \pi)^{d}} e^{i k \cdot\left(x_{1}-x_{2}\right)}\left(\frac{4}{k^{2}}\right)^{\frac{i \nu+i \bar{\nu}}{2}} \int \frac{d z}{z} \frac{d s d t}{s t} z^{\epsilon} s^{-i \nu} t^{-i \bar{\nu}} e^{-z\left(s+t+\frac{1}{s}+\frac{1}{t}\right)},
$$

where the dependence on $k$ was brought into an explicit form by doing a rescaling $s \rightarrow \frac{s k^{2}}{4 z}$, $t \rightarrow \frac{t k^{2}}{4 z}$ and $z \rightarrow \frac{2 z}{k}$. The second integral in (D.14) is

$$
\begin{aligned}
& \int \frac{d z}{z} \frac{d s d t}{s t} z^{\epsilon} s^{-i \nu} t^{-i \bar{\nu}} e^{-z\left(s+t+\frac{1}{s}+\frac{1}{t}\right)}=2 \Gamma(\epsilon) \int_{-\infty}^{\infty} d U d V \frac{e^{-i U(\nu+\bar{\nu})-i V(\nu-\bar{\nu})}}{(4 \cosh (U) \cosh (V))^{\epsilon}} \\
= & \frac{\prod_{n, m= \pm 1} \Gamma\left(\frac{\epsilon+i m \nu+i n \bar{\nu}}{2}\right)}{2 \Gamma(\epsilon)} \underset{\epsilon \rightarrow 0}{=} 2 \pi \Gamma(i \nu) \Gamma(-i \nu)[\delta(\nu-\bar{\nu})+\delta(\nu+\bar{\nu})],
\end{aligned}
$$

where $U$ and $V$ are related to $t$ and $s$ by $s=e^{U+V}$ and $t=e^{U-V}$. We conclude that the integral (D.11) over AdS of two scalar bulk-to-boudary propagators is

$$
\begin{aligned}
& \frac{2 \pi^{2 h+1} \Gamma(i \nu) \Gamma(-i \nu)}{\Gamma(h-i \nu) \Gamma(h-i \bar{\nu})} \int \frac{d^{d} k}{(2 \pi)^{d}} e^{i k \cdot\left(x_{1}-x_{2}\right)}\left(\frac{k^{2}}{4}\right)^{-\frac{i \nu+i \bar{\nu}}{2}}[\delta(\nu-\bar{\nu})+\delta(\nu+\bar{\nu})] \\
= & \frac{2 \pi^{h+1} \Gamma(-i \nu)}{\Gamma(h-i \nu)\left(x_{12}^{2}\right)^{h-i \nu}} \delta(\nu-\bar{\nu})+\frac{2 \pi^{2 h+1} \Gamma(i \nu) \Gamma(-i \nu)}{\Gamma(h+i \nu) \Gamma(h-i \nu)} \delta^{d}\left(x_{1}-x_{2}\right) \delta(\nu+\bar{\nu}) .
\end{aligned}
$$

Now we just have to act $J$ times with the differential operator $\mathcal{D}$ according to (D.10). Notice that

$$
-\frac{1}{(J+\Delta-1)(J+\Delta)} \mathcal{D} \frac{\left(H_{12}\right)^{J}}{\left(P_{1} \cdot P_{2}\right)^{\Delta}}=\frac{\left(H_{12}\right)^{J+1}}{\left(P_{1} \cdot P_{2}\right)^{\Delta}},
$$

where the structure $H_{12}$ is defined in (D.6), so $\mathcal{D}$ will generate the structure of the spin $J$ boundary two-point function,

$$
\frac{(-1)^{J}}{(\Delta-1)_{J}(\Delta)_{J}} \mathcal{D}^{J} \frac{1}{\left(-2 P_{1} \cdot P_{2}\right)^{\Delta}}=\frac{\left(H_{12}\right)^{J}}{\left(-2 P_{1} \cdot P_{2}\right)^{\Delta}} .
$$

Finally, we conclude that

$$
\begin{aligned}
& \frac{1}{J !\left(h-\frac{1}{2}\right)_{J}} \int d Y \Pi_{h-i \nu, J}\left(Y, P_{1} ; K, Z_{1}\right) \Pi_{h-i \bar{\nu}, J}\left(Y, P_{2} ; W, Z_{2}\right) \\
= & \mathcal{C}_{h-i \nu, J} \mathcal{C}_{h-i \bar{\nu}, J}\left[\delta(\nu-\bar{\nu}) \frac{2 \pi^{h+1}(h-i \nu-1) \Gamma(-i \nu)}{(h+J-i \nu-1) \Gamma(h-i \nu)} \frac{\left(H_{12}\right)^{J}}{\left(P_{12}\right)^{h-i \nu+J}}\right. \\
& \left.+\delta(\nu+\bar{\nu}) \frac{J ! 2 \pi^{2 h+1} \Gamma(i \nu) \Gamma(-i \nu)}{(h-i \nu)_{J}(h-i \bar{\nu})_{J} \Gamma(h+i \nu) \Gamma(h-i \nu)} \frac{\mathcal{D}^{J} \delta^{d}\left(P_{1}, P_{2}\right)}{\left(P_{12}\right)^{J}}\right] .
\end{aligned}
$$


There remains one integral to do on the boundary, say on the point $P_{2}$. Let us start by performing the integral of the term proportional to $\delta(\nu-\bar{\nu})$ in (D.18),

$$
\int d P_{2} \frac{\Pi_{h+i \nu, J}\left(X_{2}, P_{2} ; W_{2}, D_{Z_{2}}\right)\left(H_{12}\right)^{J}}{J !(h-1)_{J}\left(P_{12}\right)^{h-i \nu+J}} .
$$

This integrand can be written as the limit of two bulk-to-boundary propagators

$$
\int d P_{2} \frac{\Pi_{h+i \nu, J}\left(X_{2}, P_{2} ; W_{2}, D_{Z_{2}}\right)}{J !(h-1)_{J}} \frac{1}{\mathcal{C}_{h-i \nu, J}} \lim _{\substack{X_{1} \rightarrow P_{1} \\ W_{1} \rightarrow Z_{1}}} \Pi_{h-i \nu, J}\left(X_{1}, P_{2} ; W_{1}, Z_{2}\right) .
$$

If one naively takes the limit outside, the integral is proportional to the harmonic function $\Omega_{\nu, J}\left(X_{1}, X_{2} ; W_{1}, W_{2}\right)$, which itself can be written as a sum of two bulk-to-bulk propagators as in (4.3). Then the limit $X_{1} \rightarrow P_{1}$ of the bulk-to-bulk propagators just gives a sum of two bulk-to-boundary propagators from $X_{2}$ to $P_{1}$ with dimension $h+i \nu$ and $h-i \nu$. However, this cannot be correct because the original integral (D.19) had dimension $h-i \nu$ at point $P_{1}$. Dropping the term with wrong dimension, one obtains the result ${ }^{10}$

$$
\int d P_{2} \frac{\Pi_{h+i \nu, J}\left(X_{2}, P_{2} ; W_{2}, D_{Z_{2}}\right)\left(H_{12}\right)^{J}}{J !(h-1)_{J}\left(P_{12}\right)^{h-i \nu+J}}=-\frac{i}{2 \nu \mathcal{C}_{h-i \nu, J}} \Pi_{h-i \nu, J}\left(X_{2}, P_{1} ; W_{2}, Z_{1}\right) .
$$

The contribution from the term proportional to $\delta(\nu+\bar{\nu})$ in (D.18) can be easily fixed using a simple symmetry argument, since the original integral (D.1) is an even function of $\bar{\nu}$. Thus, we conclude that (D.1) is given by

$$
\frac{[\delta(\nu+\bar{\nu})+\delta(\nu-\bar{\nu})] \nu^{2}}{2 \pi J !(h-1)_{J}} \int d P_{1} \Pi_{h+i \nu, J}\left(X_{1}, P_{1} ; W_{1}, D_{Z_{1}}\right) \Pi_{h-i \nu, J}\left(X_{2}, P_{1} ; W_{2}, Z_{1}\right),
$$

which shows (4.7).

Completeness. The goal of this section is to determine the coefficients $c_{J, l}(\nu)$ that appear in the completeness relation (4.11),

$$
\sum_{l=0}^{J} \int d \nu c_{J, l}(\nu)\left(\left(W_{1} \cdot \nabla_{1}\right)\left(W_{2} \cdot \nabla_{2}\right)\right)^{l} \Omega_{\nu, J-l}\left(X_{1}, X_{2} ; W_{1}, W_{2}\right)=\delta\left(X_{1}, X_{2}\right)\left(W_{12}\right)^{J} .
$$

Our strategy will be to find a recursion relation that fixes all coefficients $c_{J, l}(\nu)$ starting from the initial condition $c_{J, 0}(\nu)=1$ derived in the main text. With this in mind, we take the divergence of the equation above at the point $X_{1}$. On the right hand side we use ${ }^{11}$

$$
\nabla_{1} \cdot K_{1}\left[\delta\left(X_{1}, X_{2}\right)\left(W_{12}\right)^{J}\right]=-\frac{J(2 h+2 J-3)}{2} W_{2} \cdot \nabla_{2}\left[\delta\left(X_{1}, X_{2}\right)\left(W_{12}\right)^{J-1}\right],
$$

and on the left hand side we use the commutation relations

$$
\begin{aligned}
{[\nabla \cdot K, W \cdot \nabla] } & =\left(\frac{2 h-1}{2}+\mathcal{D}_{W}\right) \nabla^{2}-\left(\left(\mathcal{D}_{W}\right)^{2}+\frac{3(2 h-1)}{2} \mathcal{D}_{W}+\frac{(2 h-1)^{2}}{2}\right) \mathcal{D}_{W} \\
{\left[\nabla^{2}, W \cdot \nabla\right] } & =-2\left(h-1+\mathcal{D}_{W}\right) W \cdot \nabla
\end{aligned}
$$

\footnotetext{
${ }^{10}$ The extra term was generated when we naively interchanged the limit with the integration symbol.

${ }^{11}$ To derive this formula one needs to use $W_{1} \cdot X_{2} \delta\left(X_{1}, X_{2}\right)=0$ and similar identities.
} 
where $\mathcal{D}_{W}=W \cdot \partial_{W}$. Using these basic commutators, one can show that

$$
\left[\nabla^{2},\left(W \cdot \nabla_{X}\right)^{n}\right]=-n\left(2 h-1+2 \mathcal{D}_{W}-n\right)\left(W \cdot \nabla_{X}\right)^{n} .
$$

Similarly, with a bit more effort, one finds

$$
\begin{aligned}
{\left[\nabla \cdot K,\left(W \cdot \nabla_{X}\right)^{l}\right]=} & \frac{1}{2}\left(W \cdot \nabla_{X}\right)^{l-1} l\left(2 h+l+2 \mathcal{D}_{W}-2\right) \\
& \times\left[1-l-\left(l+\mathcal{D}_{W}-1\right)\left(2 h+l+\mathcal{D}_{W}-2\right)+\nabla^{2}\right] .
\end{aligned}
$$

Using these commutators, the divergence of equation (4.11) can be written as

$$
\begin{aligned}
& -W_{2} \cdot \nabla_{2} \sum_{l=1}^{J} \int d \nu c_{J, l}(\nu) \frac{(l+1)(3-l-2 h-2 J)\left[(h+J-1)^{2}+\nu^{2}\right]}{2} \\
& \times\left(\left(W_{1} \cdot \nabla_{1}\right)\left(W_{2} \cdot \nabla_{2}\right)\right)^{l-1} \Omega_{\nu, J-l}\left(X_{1}, X_{2} ; W_{1}, W_{2}\right)=\frac{J(2 h+2 J-3)}{2} W_{2} \cdot \nabla_{2}\left[\delta\left(X_{1}, X_{2}\right) W_{12}^{J-1}\right] .
\end{aligned}
$$

Shifting the summation variable $l \rightarrow l+1$, we can identify this equation as $W_{2} \cdot \nabla_{2}$ acting on (4.11) with $J$ replaced by $J-1$. This gives the recursion relation

$$
c_{J, l+1}(\nu)=\frac{J(2 h+2 J-3)}{(l+1)(2 h+2 J-l-3)} \frac{c_{J-1, l}(\nu)}{(h+J-1)^{2}+\nu^{2}},
$$

which supplemented with the initial condition $c_{J, 0}(\nu)=1$ determines all $c_{J, l}(\nu)$. In fact, one can write the general solution in closed form,

$$
c_{J, l}(\nu)=\frac{2^{l}(J-l+1)_{l}\left(h+J-l-\frac{1}{2}\right)_{l}}{l !(2 h+2 J-2 l-1)_{l}(h+J-l-i \nu)_{l}(h+J-l+i \nu)_{l}} .
$$

\section{E Computation of partial amplitude}

The goal of this appendix is to derive the expression for the function $\alpha_{l}(\nu)$ appearing in the partial amplitude (6.7). The tensor operations present in (6.6) are of the form

$$
\begin{aligned}
& \left(W_{1} \cdot \nabla_{1}\right)^{J-l} \Pi_{\Delta, l}=\mathcal{C}_{\Delta, l}\left(W_{1} \cdot \nabla_{1}\right)^{J-l} \frac{\left(2\left(W_{1} \cdot Z\right)\left(P_{5} \cdot X_{1}\right)-2\left(W_{1} \cdot P_{5}\right)\left(Z \cdot X_{1}\right)\right)^{l}}{\left(-2 P_{5} \cdot X_{1}\right)^{\Delta+l}} \\
= & \mathcal{C}_{\Delta, l} \frac{\left(2 P_{5} \cdot W_{1}\right)^{J-l}\left(2\left(W_{1} \cdot Z\right)\left(P_{5} \cdot X_{1}\right)-\left(W_{1} \cdot P_{5}\right)\left(Z \cdot X_{1}\right)\right)^{l}(\Delta+l)_{J-l}}{\left(-2 P_{5} \cdot X_{1}\right)^{\Delta+J}} \\
& \left(W_{1} \cdot \nabla_{1}\right)^{J} \Pi_{\Delta_{2}}=\frac{\mathcal{C}_{\Delta_{2}}\left(W_{1} \cdot \nabla_{1}\right)^{J}}{\left(-2 P_{2} \cdot X_{1}\right)^{\Delta_{2}}}=\mathcal{C}_{\Delta_{2}}\left(\Delta_{2}\right)_{J} \frac{\left(2 W_{1} \cdot P_{2}\right)^{J}}{\left(-2 P_{2} \cdot X_{1}\right)^{\Delta_{2}+J}}
\end{aligned}
$$

and

$$
\begin{aligned}
(\star) & \equiv \frac{(K \cdot \nabla)^{J} \Pi_{\Delta_{2}}(W \cdot \nabla)^{J-l} \Pi_{\Delta, l}}{\mathcal{C}_{\Delta_{2}} \mathcal{C}_{\Delta, l} J !\left(h-\frac{1}{2}\right)_{J}}= \\
= & \left(\Delta_{2}\right)_{J}(\Delta+l)_{J-l} 2^{J}(J-l) ! \Gamma(3 / 2-h-J) \frac{\left(2\left(P_{2} \cdot P_{5}\right)\left(X_{1} \cdot Z\right)-2\left(P_{2} \cdot Z\right)\left(P_{5} \cdot X_{1}\right)\right)^{l}}{\left(-2 P_{2} \cdot X_{1}\right)^{\Delta_{2}+J}\left(-2 P_{5} \cdot X_{1}\right)^{\Delta+J}} \\
& \times \sum_{m=0}^{J-l} \frac{\Gamma(1-h-J+m)\left(2\left(P_{2} \cdot X_{1}\right)\left(P_{5} \cdot X_{1}\right)\right)^{m}\left(P_{25}\right)^{J-l-m}}{\Gamma\left(1-h-J+\frac{m}{2}\right) \Gamma\left(\frac{3-2 h-2 J+m}{2}\right) m !(J-l-m) !} .
\end{aligned}
$$


The first two operations follow almost immediately from the definition, just notice that in (E.1) the differential operator can act only in the denominator as it gives zero once it acts on the numerator. To obtain (E.3) we use (B.2).

The function $F_{\nu, J}$ is defined as the integral over the boundary of three-point functions. So, to derive $F_{\nu, J}$ from (6.6) we need to integrate over one of the bulk points, say $X_{1}$, generating a structure that has the form of a three-point function at points $P_{1}, P_{2}$ and $P_{5}$. Joining all the pieces that connect to the bulk point $X_{1}$, we have

$$
\int d X_{1} \Pi_{\Delta_{1}}(\star) \mathcal{C}_{\Delta_{2}} \mathcal{C}_{\Delta, l}=\frac{\mathcal{B}_{\Delta_{1}, \Delta_{2}, \Delta, l, J}\left(\left(Z \cdot P_{2}\right)\left(P_{1} \cdot P_{5}\right)-\left(Z \cdot P_{1}\right)\left(P_{2} \cdot P_{5}\right)\right)^{l}}{P_{12^{\frac{\Delta_{2}+l+\Delta_{1}-\Delta}{2}}}^{\Delta^{\Delta_{1}+\Delta+l-\Delta_{2}}} P_{15}^{2}} P_{25}^{\frac{\Delta+\Delta_{2}+l-\Delta_{1}}{2}},
$$

where the function $\mathcal{B}_{\Delta_{1}, \Delta_{2}, \Delta, l, J}$ is given by

$$
\begin{aligned}
\mathcal{B}_{\Delta_{1}, \Delta_{2}, \Delta, l, J}= & \sum_{m=0}^{J-l} \frac{\mathcal{C}_{\Delta_{2}} \mathcal{C}_{\Delta, l}\left(\Delta_{2}\right)_{J}(\Delta+l)_{J-l}(-1)^{J+m}(J-l) ! 2^{1-2 h-J}}{\sqrt{\pi}(J-l-m) ! m ! \mathcal{C}_{\Delta+J-m-l}} \\
& \times \frac{\Gamma\left(\frac{3-2 h-2 J}{2}\right)(m-h-J) !\left(\frac{\Delta+\Delta_{1}-\Delta_{2}-l}{2}\right)_{l} b_{\Delta_{1}, \Delta_{2}+J-m, \Delta+J-m-l, 0}}{\Gamma(m+2-2 h-2 J)(\Delta+J-m-l)_{l} \mathcal{C}_{\Delta_{2}+J-m}} .
\end{aligned}
$$

Notice that to perform the integration over $X_{1}$ we used an identity similar to (5.6). The function $b_{\Delta_{1}, \Delta_{2}, \Delta, J}$ is the same as in (5.7). The integration over $X_{2}$ produces a similar term and so the next step to read off the relation is to integrate the product of two three-point functions over the boundary point.

At this point we just need to bring the expression close to equation (3.16) of [62] that is related to the function $F_{\nu, J}$. So we just need to evaluate

$$
\int d P_{5} \frac{\left(P_{25}\left(D_{Z} \cdot P_{1}\right)-P_{15}\left(D_{Z} \cdot P_{2}\right)\right)^{J}}{P_{15}^{\frac{\Delta_{1}+\Delta-\Delta_{2}+J}{2}} P_{25}^{\frac{\Delta_{2}+\Delta-\Delta_{1}+J}{2}} P_{12}^{\frac{\Delta_{1}+\Delta_{2}-\Delta+J}{2}}} \frac{\left(P_{45}\left(Z \cdot P_{3}\right)-P_{35}\left(Z \cdot P_{4}\right)\right)^{J}}{P_{35}^{\frac{\Delta_{3}+d-\Delta-\Delta_{4}+J}{2}}} P_{45}^{\frac{\Delta_{4}+d-\Delta-\Delta_{3}+J}{2}} P_{34}^{\frac{\Delta_{3}+\Delta_{4}-d+\Delta+J}{2}},
$$

where the operator $D_{Z}$ is the projection operator $(4.2)$, as defined in $[11,13]$. Its action produces a Gegenbauer polynomial. The integral over $P_{5}$, involving these polynomials, is precisely (3.16) of [62], where it was shown to be equal to a linear combination of conformal blocks. Taking into account the definition of the function $F_{\nu, J}$ in terms of conformal blocks, ${ }^{12}$ it is possible to extract the coefficient $\beta$,

$$
\begin{aligned}
\beta_{\nu, \Delta_{i}, J}= & \frac{2^{3-2 J} \pi^{1+h} \Gamma(i \nu) \Gamma(-i \nu)(h-i \nu-1)_{J}(h+i \nu-1)_{J}}{\Gamma\left(\frac{\Delta_{1}+\Delta_{2}-h-i \nu+J}{2}\right) \Gamma\left(\frac{h+J+\Delta_{1}-\Delta_{2}+i \nu}{2}\right) \Gamma\left(\frac{h+J-\Delta_{1}+\Delta_{2}+i \nu}{2}\right) \Gamma\left(\frac{\Delta_{1}+\Delta_{2}+J+i \nu-h}{2}\right)} \\
& \times \frac{1}{\Gamma\left(\frac{h+J+\Delta_{3}-\Delta_{4}-i \nu}{2}\right) \Gamma\left(\frac{h+J+\Delta_{4}-\Delta_{3}-i \nu}{2}\right) \Gamma\left(\frac{\Delta_{3}+\Delta_{4}+J-h-i \nu}{2}\right) \Gamma\left(\frac{\Delta_{3}+\Delta_{4}+i \nu-h+J}{2}\right)} .
\end{aligned}
$$

\footnotetext{
${ }^{12}$ Notice that the conformal blocks of [62] and [53] have different normalization.
} 
The coefficient $\alpha_{l}(\nu)$ can be read after gathering all the components together,

$$
\alpha_{l}=\mathcal{B}_{\Delta_{1}, \Delta_{2}, h+i \nu, l, J} \mathcal{B}_{\Delta_{3}, \Delta_{4}, h-i \nu, l, J} \frac{\nu^{2} \beta_{\nu, \Delta_{i}, l}}{\pi J !(h-1)_{J}},
$$

or explicitly,

$$
\alpha_{l}(\nu)=\frac{((J-l) !)^{2} \Gamma^{2}\left(\frac{3-2 h-2 J}{2}\right) R_{J, l}\left(\nu, \Delta_{1}, \Delta_{2}\right) R_{J, l}\left(-\nu, \Delta_{3}, \Delta_{4}\right)}{\pi^{3 h+1} 2^{4 h+4 J+2 l+3} \Gamma\left(\Delta_{1}+1-h\right) \Gamma\left(\Delta_{2}+1-h\right) \Gamma\left(\Delta_{3}+1-h\right) \Gamma\left(\Delta_{4}+1-h\right)},
$$

where we defined

$$
\begin{aligned}
R_{J, l}\left(\nu, \Delta_{1}, \Delta_{2}\right)=\sum_{p=0}^{J-l} & \frac{(-1)^{p} \Gamma(p+1-h-J)(h+J-p+i \nu)_{p}\left(J-p+\Delta_{2}\right)_{p}}{(J-l-p) ! p ! \Gamma(p+2-2 h-2 J)} \\
& \times\left(\frac{\Delta_{1}+\Delta_{2}-h+l+i \nu}{2}\right)_{J-l-p}\left(\frac{h+l+i \nu-\Delta_{12}}{2}\right)_{J-l-p} .
\end{aligned}
$$

Open Access. This article is distributed under the terms of the Creative Commons Attribution License (CC-BY 4.0), which permits any use, distribution and reproduction in any medium, provided the original author(s) and source are credited.

\section{References}

[1] J.M. Maldacena, The Large-N limit of superconformal field theories and supergravity, Int. J. Theor. Phys. 38 (1999) 1113 [hep-th/9711200] [INSPIRE].

[2] M.A. Vasiliev, Consistent equation for interacting gauge fields of all spins in (3+1)-dimensions, Phys. Lett. B 243 (1990) 378 [INSPIRE].

[3] M.A. Vasiliev, More on equations of motion for interacting massless fields of all spins in (3+1)-dimensions, Phys. Lett. B 285 (1992) 225 [INSPIRE].

[4] M.A. Vasiliev, Higher spin gauge theories in four-dimensions, three-dimensions and two-dimensions, Int. J. Mod. Phys. D 5 (1996) 763 [hep-th/9611024] [InSPIRE].

[5] M.A. Vasiliev, Higher spin gauge theories: Star product and AdS space, hep-th/9910096 [INSPIRE].

[6] I.R. Klebanov and A.M. Polyakov, AdS dual of the critical $O(N)$ vector model, Phys. Lett. B 550 (2002) 213 [hep-th/0210114] [INSPIRE].

[7] E. Sezgin and P. Sundell, Holography in $4 D$ (super) higher spin theories and a test via cubic scalar couplings, JHEP 07 (2005) 044 [hep-th/0305040] [INSPIRE].

[8] R.G. Leigh and A.C. Petkou, Holography of the $N=1$ higher spin theory on AdS $S_{4}, J H E P 06$ (2003) 011 [hep-th/0304217] [INSPIRE].

[9] L. Cornalba, M.S. Costa and J. Penedones, Deep Inelastic Scattering in Conformal QCD, JHEP 03 (2010) 133 [arXiv:0911.0043] [INSPIRE].

[10] S. Weinberg, Six-dimensional Methods for Four-dimensional Conformal Field Theories, Phys. Rev. D 82 (2010) 045031 [arXiv: 1006.3480] [InSPIRE]. 
[11] M.S. Costa, J. Penedones, D. Poland and S. Rychkov, Spinning Conformal Correlators, JHEP 11 (2011) 071 [arXiv: 1107.3554] [INSPIRE].

[12] A. Dymarsky, On the four-point function of the stress-energy tensors in a CFT, arXiv: 1311.4546 [INSPIRE].

[13] M.S. Costa, J. Penedones, D. Poland and S. Rychkov, Spinning Conformal Blocks, JHEP 11 (2011) 154 [arXiv:1109.6321] [INSPIRE].

[14] D. Simmons-Duffin, Projectors, Shadows and Conformal Blocks, JHEP 04 (2014) 146 [arXiv: 1204.3894] [INSPIRE].

[15] J. Penedones, Writing CFT correlation functions as AdS scattering amplitudes, JHEP 03 (2011) 025 [arXiv:1011.1485] [INSPIRE].

[16] M.F. Paulos, Towards Feynman rules for Mellin amplitudes, JHEP 10 (2011) 074 [arXiv:1107.1504] [INSPIRE].

[17] A.L. Fitzpatrick, J. Kaplan, J. Penedones, S. Raju and B.C. van Rees, A Natural Language for AdS/CFT Correlators, JHEP 11 (2011) 095 [arXiv: 1107.1499] [INSPIRE].

[18] A. Naqvi, Propagators for massive symmetric tensor and $p$ forms in AdS(d+1), JHEP 12 (1999) 025 [hep-th/9911182] [INSPIRE].

[19] S. Giombi, I.R. Klebanov, S.S. Pufu, B.R. Safdi and G. Tarnopolsky, AdS Description of Induced Higher-Spin Gauge Theory, JHEP 10 (2013) 016 [arXiv:1306.5242] [INSPIRE].

[20] E. D'Hoker, D.Z. Freedman, S.D. Mathur, A. Matusis and L. Rastelli, Graviton and gauge boson propagators in AdS(d+1), Nucl. Phys. B 562 (1999) 330 [hep-th/9902042] [INSPIRE].

[21] T. Leonhardt, R. Manvelyan and W. Rühl, The Group approach to AdS space propagators, Nucl. Phys. B 667 (2003) 413 [hep-th/0305235] [inSPIRE].

[22] T. Leonhardt, W. Rühl and R. Manvelyan, The Group approach to AdS space propagators: A Fast algorithm, J. Phys. A 37 (2004) 7051 [hep-th/0310063] [INSPIRE].

[23] I. Balitsky, Mellin representation of the graviton bulk-to-bulk propagator in AdS, Phys. Rev. D 83 (2011) 087901 [arXiv:1102.0577] [INSPIRE].

[24] G. Giecold, A Universal Formula for the Stress-Tensor Contribution to Scalar Four-Point Functions, Phys. Rev. D 86 (2012) 106003 [arXiv:1205.6444] [INSPIRE].

[25] J. Penedones, High Energy Scattering in the AdS/CFT Correspondence, arXiv:0712.0802 [INSPIRE].

[26] L. Cornalba, M.S. Costa, J. Penedones and R. Schiappa, Eikonal Approximation in AdS/CFT: From Shock Waves to Four-Point Functions, JHEP 08 (2007) 019 [hep-th/0611122] [INSPIRE].

[27] R.R. Metsaev, Massless mixed symmetry bosonic free fields in d-dimensional anti-de Sitter space-time, Phys. Lett. B 354 (1995) 78 [INSPIRE].

[28] E. Joung and M. Taronna, Cubic interactions of massless higher spins in (A)dS: metric-like approach, Nucl. Phys. B 861 (2012) 145 [arXiv:1110.5918] [INSPIRE].

[29] A. Cap and A.R. Gover, Standard tractors and the conformal ambient metric construction, Annals Global Anal. Geom. 24 (2003) 231 [math/0207016] [INSPIRE].

[30] A.R. Gover, A. Shaukat and A. Waldron, Tractors, Mass and Weyl Invariance, Nucl. Phys. B 812 (2009) 424 [arXiv:0810.2867] [INSPIRE]. 
[31] M. Fierz and W. Pauli, On relativistic wave equations for particles of arbitrary spin in an electromagnetic field, Proc. Roy. Soc. Lond. A 173 (1939) 211.

[32] A. Higuchi, Forbidden Mass Range for Spin-2 Field Theory in de Sitter Space-time, Nucl. Phys. B 282 (1987) 397 [INSPIRE].

[33] N. Bouatta, G. Compere and A. Sagnotti, An Introduction to free higher-spin fields, hep-th/0409068 [INSPIRE].

[34] D. Francia, Geometric Lagrangians for massive higher-spin fields, Nucl. Phys. B 796 (2008) 77 [arXiv: 0710.5378] [inSPIRE].

[35] D. Francia, On the Relation between Local and Geometric Lagrangians for Higher spins, J. Phys. Conf. Ser. 222 (2010) 012002 [arXiv: 1001. 3854] [INSPIRE].

[36] B. Allen and T. Jacobson, Vector Two Point Functions in Maximally Symmetric Spaces, Commun. Math. Phys. 103 (1986) 669 [inSPIRE].

[37] K. Mkrtchyan, Higher Spin Interacting Quantum Field Theory and Higher Order Conformal Invariant Lagrangians, arXiv:1011.0160 [INSPIRE].

[38] D. Francia, J. Mourad and A. Sagnotti, (A)dS exchanges and partially-massless higher spins, Nucl. Phys. B 804 (2008) 383 [arXiv:0803.3832] [InSPIRE].

[39] L.P.S. Singh and C.R. Hagen, Lagrangian formulation for arbitrary spin. 1. The boson case, Phys. Rev. D 9 (1974) 898 [inSPIRE].

[40] S.D. Rindani and M. Sivakumar, Gauge - Invariant Description of Massive Higher - Spin Particles by Dimensional Reduction, Phys. Rev. D 32 (1985) 3238 [InSPIRE].

[41] C. Aragone, S. Deser and Z. Yang, Massive Higher Spin From Dimensional Reduction of Gauge Fields, Annals Phys. 179 (1987) 76 [INSPIRE].

[42] I.I. Kogan, S. Mouslopoulos and A. Papazoglou, The $m \rightarrow 0$ limit for massive graviton in $d S(4)$ and $A d S_{4}$ : How to circumvent the van Dam-Veltman-Zakharov discontinuity, Phys. Lett. B 503 (2001) 173 [hep-th/0011138] [INSPIRE].

[43] M. Porrati, No van Dam-Veltman-Zakharov discontinuity in AdS space, Phys. Lett. B 498 (2001) 92 [hep-th/0011152] [INSPIRE].

[44] D.Z. Freedman, S.D. Mathur, A. Matusis and L. Rastelli, Correlation functions in the CFT $(d) / A d S(d+1)$ correspondence, Nucl. Phys. B 546 (1999) 96 [hep-th/9804058] [INSPIRE].

[45] L. Cornalba, M.S. Costa and J. Penedones, Eikonal Methods in AdS/CFT: BFKL Pomeron at Weak Coupling, JHEP 06 (2008) 048 [arXiv:0801.3002] [INSPIRE].

[46] H. Osborn and A.C. Petkou, Implications of conformal invariance in field theories for general dimensions, Annals Phys. 231 (1994) 311 [hep-th/9307010] [INSPIRE].

[47] F.A. Dolan and H. Osborn, Conformal four point functions and the operator product expansion, Nucl. Phys. B 599 (2001) 459 [hep-th/0011040] [INSPIRE].

[48] H. Liu and A.A. Tseytlin, $D=4$ super Yang-Mills, $D=5$ gauged supergravity and $D=4$ conformal supergravity, Nucl. Phys. B 533 (1998) 88 [hep-th/9804083] [INSPIRE].

[49] P. Kovtun and A. Ritz, Black holes and universality classes of critical points, Phys. Rev. Lett. 100 (2008) 171606 [arXiv:0801.2785] [INSPIRE]. 
[50] T. Bargheer, J.A. Minahan and R. Pereira, Computing Three-Point Functions for Short Operators, JHEP 03 (2014) 096 [arXiv:1311.7461] [INSPIRE].

[51] L.F. Alday and A. Bissi, Higher-spin correlators, JHEP 10 (2013) 202 [arXiv:1305.4604] [INSPIRE].

[52] V.K. Dobrev, V.B. Petkova, S.G. Petrova and I.T. Todorov, Dynamical Derivation of Vacuum Operator Product Expansion in Euclidean Conformal Quantum Field Theory, Phys. Rev. D 13 (1976) 887 [INSPIRE].

[53] M.S. Costa, V. Goncalves and J. Penedones, Conformal Regge theory, JHEP 12 (2012) 091 [arXiv: 1209.4355] [INSPIRE].

[54] E. D'Hoker, D.Z. Freedman, S.D. Mathur, A. Matusis and L. Rastelli, Graviton exchange and complete four point functions in the AdS/CFT correspondence, Nucl. Phys. B 562 (1999) 353 [hep-th/9903196] [INSPIRE].

[55] G. Mack, D-independent representation of Conformal Field Theories in D dimensions via transformation to auxiliary Dual Resonance Models. Scalar amplitudes, arXiv:0907.2407 [INSPIRE].

[56] B.M. Barker, S.N. Gupta and R.D. Haracz, One-Graviton Exchange Interaction of Elementary Particles, Phys. Rev. 149 (1966) 1027 [INSPIRE].

[57] S.R. Huggins and D.J. Toms, One Graviton Exchange Interaction of Nonminimally Coupled Scalar Fields, Class. Quant. Grav. 4 (1987) 1509 [INSPIRE].

[58] L. Cornalba, M.S. Costa and J. Penedones, Eikonal approximation in AdS/CFT: Resumming the gravitational loop expansion, JHEP 09 (2007) 037 [arXiv:0707.0120] [INSPIRE].

[59] A.L. Fitzpatrick, J. Kaplan, D. Poland and D. Simmons-Duffin, The Analytic Bootstrap and AdS Superhorizon Locality, JHEP 12 (2013) 004 [arXiv:1212.3616] [INSPIRE].

[60] Z. Komargodski and A. Zhiboedov, Convexity and Liberation at Large Spin, JHEP 11 (2013) 140 [arXiv: 1212.4103] [INSPIRE].

[61] A.L. Fitzpatrick, J. Kaplan and M.T. Walters, Universality of Long-Distance AdS Physics from the CFT Bootstrap, JHEP 08 (2014) 145 [arXiv:1403.6829] [INSPIRE].

[62] F.A. Dolan and H. Osborn, Conformal Partial Waves: Further Mathematical Results, arXiv:1108.6194 [INSPIRE]. 